\title{
All-Cause Mortality and Cardiovascular Death between Statins and Omega-3 Supplementation: A Meta-Analysis and Network Meta-Analysis from 55 Randomized Controlled Trials
}

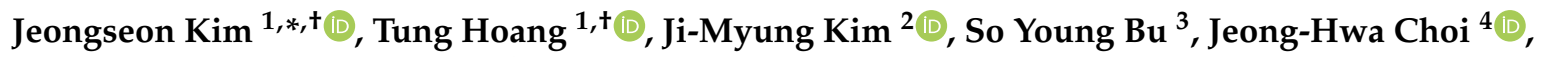 \\ Eunju Park ${ }^{5}$, Seung-Min Lee ${ }^{6}$ D, Eunmi Park ${ }^{7}$, Ji Yeon Min ${ }^{8}$, In Seok Lee ${ }^{9}$, So Young Youn ${ }^{10}$ \\ and Jee-Young Yeon ${ }^{11}$ \\ 1 Department of Cancer Biomedical Science, National Cancer Center Graduate School of Cancer Science and \\ Policy, Goyang 10408, Korea; 75256@ncc.re.kr \\ 2 Food and Nutrition Major, Division of Food Science and Culinary Arts, Shinhan University, \\ Uijeongbu 11644, Korea; doublekim@hanmail.net \\ 3 Department of Food and Nutrition, Daegu University, Gyeongsan 38453, Korea; busy@daegu.ac.kr \\ 4 Department of Food and Nutrition, Keimyung University, Daegu 42601, Korea; jhchoi@kmu.ac.kr \\ 5 Department of Food and Nutrition, Kyungnam University, Changwon 51767, Korea; pej@kyungnam.ac.kr \\ 6 Department of Food and Nutrition, Yonsei University, Seoul 03722, Korea; leeseungmin@yonsei.ac.kr \\ 7 Department of Food and Nutrition, Hannam University, Daejeon 34430, Korea; eunmi_park@hnu.kr \\ 8 Dietetics and Nutrition Services Team, Asan Medical Center, Seoul 05505, Korea; sophiajym@hanmail.net \\ 9 Nutrition Support Team, Kyung Hee University Medical Center, Seoul 02447, Korea; inseok77@gmail.com \\ 10 Clinical Nutrition Part, Samsung Medical Center, Seoul 06351, Korea; soyoungy.youn@samsung.com \\ 11 Department of Food and Nutrition, Seowon University, Cheongju 28674, Korea; yeon_fn@naver.com \\ * Correspondence: jskim@ncc.re.kr; Tel.: +82-31-920-2570 \\ + These authors contributed equally to this work.
}

Received: 24 September 2020; Accepted: 19 October 2020; Published: 20 October 2020

\begin{abstract}
Statins and omega-3 supplementation have shown potential benefits in preventing cardiovascular disease (CVD), but their comparative effects on mortality outcomes, in addition to primary and secondary prevention and mixed population, have not been investigated. This study aimed to examine the effect of statins and omega-3 supplementation and indirectly compare the effects of statin use and omega-3 fatty acids on all-cause mortality and CVD death. We included randomized controlled trials (RCTs) from meta-analyses published until December 2019. Pooled relative risks (RRs) and 95\% confidence intervals (CIs) were calculated to indirectly compare the effect of statin use versus omega- 3 supplementation in a frequentist network meta-analysis. In total, 55 RCTs were included in the final analysis. Compared with placebo, statins were significantly associated with a decreased the risk of all-cause mortality $(R R=0.90,95 \% C I=0.86-0.94)$ and CVD death $(R R=0.86$, $95 \% \mathrm{CI}=0.80-0.92$ ), while omega-3 supplementation showed a borderline effect on all-cause mortality $(\mathrm{RR}=0.97,95 \% \mathrm{CI}=0.94-1.01)$ but were significantly associated with a reduced risk of CVD death $(\mathrm{RR}=0.92,95 \% \mathrm{CI}=0.87-0.98)$ in the meta-analysis. The network meta-analysis found that all-cause mortality was significantly different between statin use and omega-3 supplementation for overall population ( $R R=0.91,95 \% \mathrm{CI}=0.85-0.98$ ), but borderline for primary prevention and mixed population and nonsignificant for secondary prevention. Furthermore, there were borderline differences between statin use and omega-3 supplementation in CVD death in the total population $(R R=0.92,95 \% C I=0.82-1.04)$ and primary prevention $(R R=0.85,95 \% C I=0.68-1.05)$, but nonsignificant differences in secondary prevention $(\mathrm{RR}=0.97,95 \% \mathrm{CI}=0.66-1.43)$ and mixed population ( $R R=0.92,95 \% \mathrm{CI}=0.75-1.14)$. To summarize, statin use might be associated with a lower risk of all-cause mortality than omega-3 supplementation. Future direct comparisons between statin use and omega-3 supplementation are required to confirm the findings.
\end{abstract}


Keywords: cardiovascular disease; mortality; statin; omega-3; network meta-analysis

\section{Introduction}

According to the Global Burden of Diseases 2017, an estimated 17.3 million (31\%) deaths worldwide were caused by cardiovascular disease (CVD) [1]. It has been estimated that 125.6 million Americans will have CVD by 2030, an approximately $14 \%$ increase in prevalence from prior estimates in 2020 [2]. Although life expectancy increased by 7.4 years from 1990 to 2017 [3] and survival from CVD improved by $10.3 \%$ during $2007-2017$ [1], the five-year mortality due to CVD remains as high as $13.7 \%$ in some age groups and populations [4].

To reduce CVD risk, lipid-lowering therapy such as statins has been recommended for the primary and secondary prevention of CVD [5-7]. Epidemiological evidence showed significantly reduced mortality-both from any cause and from CVD mortality-in statin-treated patients, with RRs $(95 \% \mathrm{CI})$ of $0.89(0.85-0.93)$ and $0.80(0.71-0.91)$, respectively [8]. Among patients with established CVD, statins led to a statistically significant $12 \%$ reduction in CVD deaths compared to the control group $(R R=0.88$, $95 \% \mathrm{CI}=0.81-0.96)$. However, statin-treated patients might also suffer from some harmful effects, including myopathy, diabetes, and hepatic dysfunction [8].

Furthermore, guidance on lifestyle management from the American College of Cardiology/American Heart Association (ACC/AHA) recommended a greater intake of fish or polyunsaturated fatty acids (particularly omega-3) as part of Mediterranean and Dietary Approaches to Stop Hypertension dietary patterns $[9,10]$. Omega- 3 fatty acids can also be available as supplements that contain eicosapentaenoic acid (EPA) in combination with docosahexaenoic acid (DHA) or EPA only (with either ethyl or non-ethyl structures) [11]. A recent review from the AHA did not strongly recommend omega-3 supplementation to prevent CVD [12]. Higher intake of omega-3, regardless of the source, was found to have little or no effect on all-cause mortality (relative risk (RR) $=0.98$, 95\% confidence interval $(C I)=0.93-1.03)$ and CVD death $(R R=0.94,95 \% C I=0.89-1.00)$ [13]. However, updated findings from a recent meta-analysis showed that omega-3 supplementation was associated with a $7 \%$ lower risk of CVD death $(R R=0.93,95 \% \mathrm{CI}=0.88-0.99)$ [14].

A recent study compared the effect of statins and omega-3 supplementation on risk reduction of CVD events including total CVD, coronary heart disease, myocardial infarction, and stroke [15]. However, such effects in terms of long-term outcomes such as mortality have not been elucidated. Additionally, the pooled estimates were not specified for primary and secondary prevention population and the dose-response relationship was not investigated. Therefore, we conducted this study to examine the dose-response effect of statins and omega-3 supplementation in different study population. Furthermore, given that the most recent updated evidence reported significant effects of omega-3 supplementation [14], which has fewer side effects than statins, we conducted this meta-analysis of randomized controlled trials (RCTs) to estimate the relative effects of statin use and omega-3 supplementation compared with placebo and performed a network meta-analysis (NMA) to estimate the comparative effects of statin use versus omega- 3 supplementation on all-cause mortality and CVD death.

\section{Materials and Methods}

\subsection{Search Strategy}

We used PubMed to retrieve systematic reviews with or without meta-analysis. On 22 December 2019, the following terms were searched without language restriction: statin, omega-3 supplementation, cardiovascular disease, systematic review, and meta-analysis. The RCTs from relevant systematic reviews were then assessed for eligibility criteria. We incidentally searched for relevant RCTs published after the cut-off date for inclusion in previous systematic reviews. 


\subsection{Study Eligibility}

The inclusion criteria for studies to be evaluated in the final analysis were as follows: (i) statins or omega-3 supplementation were compared with or added to a placebo; (ii) the sample size and the number of all-cause mortality and CVD death events were reported; and (iii) the follow-up duration was at least one year. RCTs were excluded if the source of omega-3 was from dietary intake or the comparison arm contained omega- 6 fatty acids.

Two investigators (T.H. and J.K.) independently assessed articles for the inclusion and exclusion criteria and were responsible for data extraction. Any discrepancies were discussed and resolved through consultations with other investigators (J.-M.K., S.Y.B, and J.-H.C.). Details were recorded about the authors' name; publication year; country; recruitment period; the mean or median follow-up time; body mass index (BMI); demographic information on age and sex; history of CVD, coronary heart disease, myocardial infarction, heart failure, hypertension, dyslipidemia, and diabetes; the mean or median number of smokers and obese subjects; sample size; the daily dose of the intervention; and the number and percentage of outcome events for each treatment arm.

\subsection{Statistical Analyses}

We conducted both direct and indirect comparisons for all-cause mortality and CVD death. In the direct comparison, we investigated the effects of statins or omega-3 supplementation compared with the placebo in a random-effects model using the DerSimonian-Laird method [16]. We additionally performed subgroup analyses by type of prevention, type of statin, and type of omega-3 supplementation. In particular, RCTs in which at least $80 \%$ of the study population had any CVD risk factors (hypertension, dyslipidemia, diabetes, smoking, or obesity) were considered to be conducted for primary prevention, whereas those in which at least $80 \%$ of the study population had any history of CVD events (CVD, coronary heart disease, myocardial infarction, and heart failure) were considered to be conducted for secondary prevention. The remaining RCTs were considered as mixed population. In the indirect comparison, pooled RRs and 95\% CIs were calculated to examine the pairwise comparisons of statins versus placebo, omega-3 supplementation versus placebo, and statins versus omega-3 supplementation in an NMA using a frequentist approach [17].

Heterogeneity across studies was measured by calculating the Higgins $\mathrm{I}^{2}$ [18]. Substantial heterogeneity was considered to be present if the $\mathrm{I}^{2}$ value was greater than $50 \%$ or the $p$-value was less than 0.05 . Evidence of asymmetry and publication bias was assessed using Begg funnel plots [19] and the Egger test [20], in line with recent recommendations [21]. Publication bias was considered to be present if the funnel plot was asymmetric or if the $p$-value from the Egger test was lower than 0.05 . In this case, pooled estimates from the fixed-effects model would be reported along with those from the random-effects model to counterweight the possible inflation of the therapeutic effect among large and small individual RCTs. For dose-response meta-analysis, the correlated RR estimates across different doses of statins and omega-3 supplementation were calculated using the generalized least-square regression method.

All statistical analyses were performed using Stata SE version 14.0 (StataCorp, College Station, TX, USA).

\section{Results}

\subsection{Literature Search}

We identified 1233 articles in the database search (Figure 1). After irrelevant records were removed, 71 full texts were screened. Of these, 61 were discarded because they addressed irrelevant topics ( $n=26)$, reported inappropriate outcomes $(n=16)$, or were duplicate or overlapping $(n=19)$. From the remaining 10 systematic reviews and meta-analyses, 374 RCTs were extracted and accessed to evaluate them for the eligibility criteria. After additionally hand-searching for updated RCTs $(n=3)$ and 
excluding ineligible RCTs ( $n=322), 55$ studies [5,22-76] with 36 RCTs of statins and 19 RCTs of omega-3 supplementation were included in the final meta-analysis and NMA.

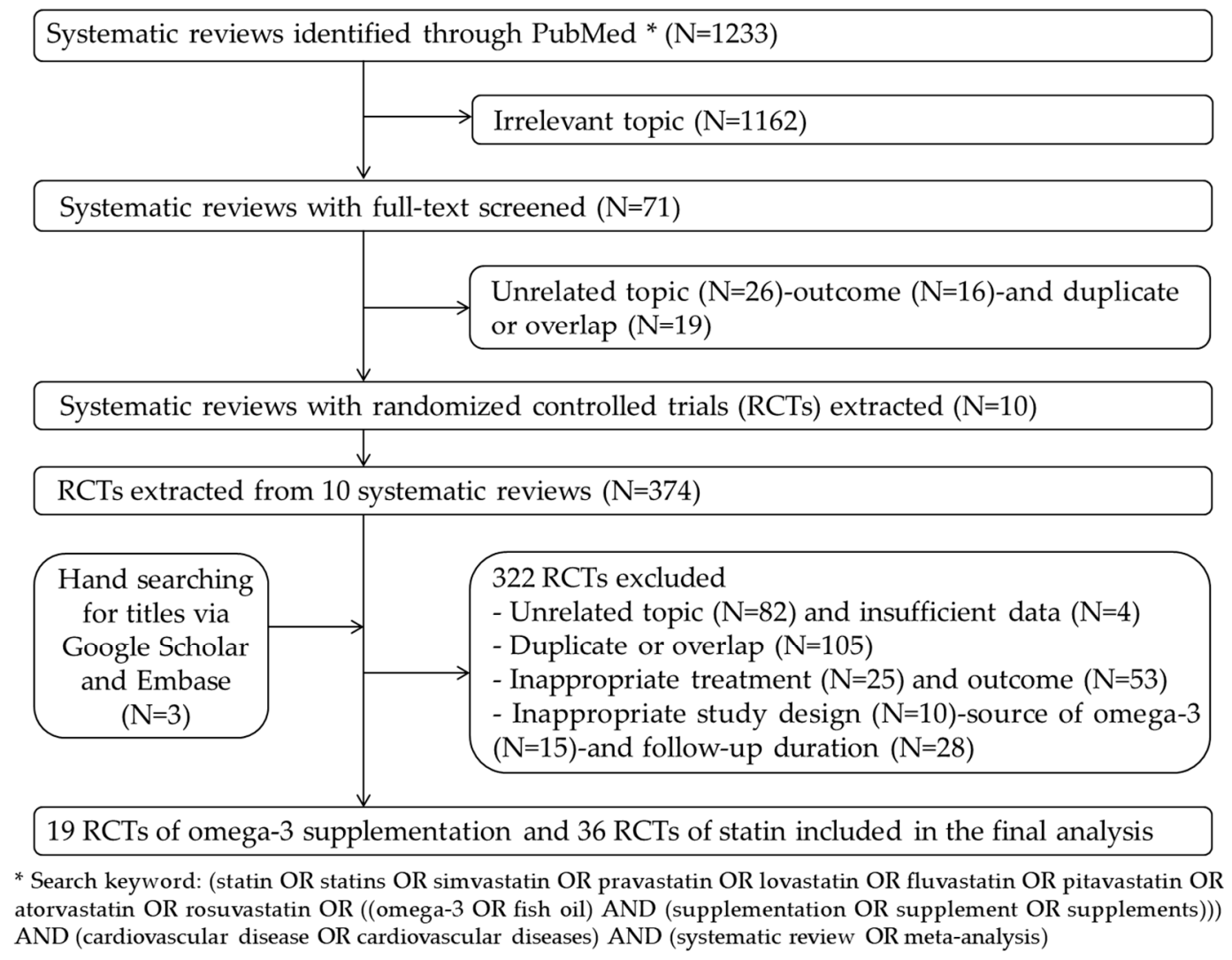

Figure 1. Flowchart of search strategy and study selection.

\subsection{Descriptive Characteristics}

Tables A1-A3 summarize the characteristics of the studies included in the final analysis. In total, 278,954 participants were assigned to receive a statin $(n=73,676)$, omega- 3 supplementation $(n=65,819)$, or a placebo $(n=139,459)$. The median age, percentage of male subjects, follow-up duration, and BMI across studies was 52.5 years old, $74.4 \%, 3.9$ years, and $27 \mathrm{~kg} / \mathrm{m}^{2}$, respectively. The following distribution was found for patients with a history of various conditions: CVD, 18.5\%; coronary heart disease, $13.0 \%$; myocardial infarction, 24.2\%; heart failure, 3.5\%; hypertension, 46.0\%; dyslipidemia, 37.5\%; diabetes, 19.2\%; smoking, 40.4\%; and obesity, 37.1\%.

In the primary prevention population, 68,101 participants were assigned to receive a statin $(n=8908)$, omega-3 supplementation $(n=25,149)$, or a placebo $(n=34,044)$. The median age, percentage of male subjects, follow-up duration, and BMI across studies were 63.15 years old, $67.15 \%$, four years, and $28.75 \mathrm{~kg} / \mathrm{m}^{2}$, respectively. The following distribution was found for patients with a history of CVD risk factors: hypertension, 55.1\%; dyslipidemia, 71.2\%; diabetes, 79.95\%; smoking, $47.1 \%$; and obesity, $47.55 \%$.

In the secondary prevention population, 19,805 participants were assigned to receive a statin $(n=3996)$, omega-3 supplementation $(n=5898)$, or a placebo $(n=9911)$. The median age, percentage of male subjects, follow-up duration, and BMI across studies were 68 years old, 78.2\%, 3.4 years, and $27 \mathrm{~kg} / \mathrm{m}^{2}$, respectively. The following distribution was found for patients with a history of CVD events: coronary heart disease, 100\%; myocardial infarction, 60\%; and heart failure, $100 \%$.

In the mixed population, 191,048 participants were assigned to receive a statin $(n=60,772)$, omega-3 supplementation $(n=34,772)$, or a placebo $(n=95,504)$. The median age, percentage of male subjects, follow-up duration, and BMI across studies were 62.05 years old, 71.2\%, 3.65 years, 
and $26.6 \mathrm{~kg} / \mathrm{m}^{2}$, respectively. The following distribution was found for patients with a history of various conditions: CVD, 18.5\%; coronary heart disease, 12.95\%; myocardial infarction, 18.85\%; heart failure, 0\%; hypertension, $41.8 \%$; dyslipidemia, 36.8\%; diabetes, $14.8 \%$; smoking, $42.2 \%$; and obesity, $20.55 \%$.

\subsection{Meta-Analysis}

Direct comparisons of statin use or omega-3 supplementation versus placebo in terms of mortality outcomes are presented in Table 1 and Figures A1-A8. Overall, statin use showed a statistically significant risk reduction for all-cause mortality and CVD death, with RRs (95\% CIs) 0.90 (0.86-0.94) and $0.86(0.80-0.92)$, respectively, whereas the findings for omega-3 supplementation were of borderline significance, with RRs (95\% CIs) of 0.97 (0.94-1.01) and 0.92 (0.87-0.98), respectively. Figure A9 shows that no publication bias was detected for the association between statin use and all-cause mortality $(p=0.22)$ or CVD death $(p=0.15)$. Similarly, no publication bias was detected for the association between omega-3 supplementation and all-cause mortality $(p=0.36)$ or CVD death $(p=0.29)$.

Table 1. Meta-analysis of the associations between statins and omega-3 supplementation with all-cause mortality and cardiovascular death.

\begin{tabular}{|c|c|c|c|c|}
\hline \multirow{2}{*}{ Intervention } & \multicolumn{2}{|c|}{ All-Cause Mortality } & \multicolumn{2}{|c|}{ Cardiovascular Disease Death } \\
\hline & No. Studies $\left(\mathrm{I}^{2}\right)$ & RR (95\% CI) & No. Studies $\left(\mathbf{I}^{2}\right)$ & RR $(95 \%$ CI) \\
\hline \multicolumn{5}{|c|}{ Statins } \\
\hline Overall & $30(43.8 \%)$ & $0.90(0.86-0.94)$ & $27(37.2 \%)$ & $0.86(0.80-0.92)$ \\
\hline Primary prevention & $5(21.8 \%)$ & $0.92(0.81-1.04)$ & $5(0 \%)$ & $0.81(0.66-1.01)$ \\
\hline Secondary prevention & $3(0 \%)$ & $0.95(0.88-1.03)$ & $3(49.4 \%)$ & $0.55(0.20-1.45)$ \\
\hline Mixed population & $21(54.1 \%)$ & $0.89(0.84-0.94)$ & $19(37.2 \%)$ & $0.90(0.73-1.12)$ \\
\hline Atorvastatin & $7(22.6 \%)$ & $0.92(0.84-1.01)$ & $5(0 \%)$ & $0.78(0.69-0.87)$ \\
\hline Pravastatin & $10(39.7 \%)$ & $0.89(0.81-0.97)$ & $8(1.0 \%)$ & $0.85(0.78-0.93)$ \\
\hline Simvastatin & $3(76.0 \%)$ & $0.85(0.66-1.09)$ & $3(19.6 \%)$ & $0.75(0.45-1.27)$ \\
\hline Rosuvastatin & $5(29.4 \%)$ & $0.96(0.90-1.01)$ & $5(0 \%)$ & $0.98(0.92-1.04)$ \\
\hline Lovastatin & $2(0 \%)$ & $0.23(0.05-1.09)$ & 1 (NA) & $0.68(0.92-1.26)$ \\
\hline Fluvastatin & $2(0 \%)$ & $0.71(0.49-1.05)$ & $4(0 \%)$ & $0.52(0.31-0.86)$ \\
\hline Pitavastatin & 1 (NA) & $0.72(0.45-1.16)$ & 1 (NA) & $0.52(0.31-0.86)$ \\
\hline \multicolumn{5}{|c|}{ Omega-3 supplementation (including REDUCE-IT trial) } \\
\hline Overall & $19(6.0 \%)$ & $0.97(0.94-1.01)$ & $14(13.4 \%)$ & $0.92(0.87-0.98)$ \\
\hline Primary prevention & $5(0 \%)$ & $1.04(0.90-1.20)$ & $4(45.4 \%)$ & $0.91(0.81-1.03)$ \\
\hline Secondary prevention & $2(0 \%)$ & $0.98(0.94-1.01)$ & $2(0 \%)$ & $0.93(0.86-1.02)$ \\
\hline Mixed population & $12(33.8 \%)$ & $1.09(0.91-1.27)$ & $8(23.3 \%)$ & $0.92(0.78-1.09)$ \\
\hline $\mathrm{EPA}+\mathrm{DHA}$ & $17(2.2 \%)$ & $0.97(0.94-1.00)$ & $13(10.0 \%)$ & $0.93(0.88-0.99)$ \\
\hline EPA only & $2(6.0 \%)$ & $0.98(0.81-1.18)$ & 1 (NA) & $0.82(0.67-0.99)$ \\
\hline \multicolumn{5}{|c|}{ Omega-3 supplementation (excluding REDUCE-IT trial) } \\
\hline Overall & $18(4.4 \%)$ & $0.98(0.95-1.01)$ & $13(10.0 \%)$ & $0.93(0.88-0.99)$ \\
\hline Primary prevention & $4(0 \%)$ & $0.98(0.93-1.04)$ & $3(42.2 \%)$ & $0.95(0.83-1.08)$ \\
\hline Secondary prevention & $2(0 \%)$ & $0.98(0.94-1.01)$ & $2(0 \%)$ & $0.93(0.86-1.02)$ \\
\hline Mixed population & $12(33.8 \%)$ & $1.09(0.91-1.27)$ & $8(23.3 \%)$ & $0.92(0.78-1.09)$ \\
\hline $\mathrm{EPA}+\mathrm{DHA}$ & $17(2.2 \%)$ & $0.97(0.94-1.00)$ & $13(10.0 \%)$ & $0.93(0.88-0.99)$ \\
\hline EPA only & $1(\mathrm{NA})$ & $1.08(0.91-1.27)$ & - & - \\
\hline
\end{tabular}

$\mathrm{RR}$, relative risk; CI, confidence interval; EPA, eicosapentaenoic acid; DHA, docosahexaenoic acid; NA, not applicable; REDUCE-IT, Reduction of Cardiovascular Events with Icosapent Ethyl - Intervention Trial. Bold font indicates statistical significance.

In the subgroup analysis by type of prevention, statins was observed to reduce all-cause mortality for the mixed population $(R R=0.89,95 \% C I=0.84-0.94)$, but borderline for primary $(R R=0.92$, $95 \% \mathrm{CI}=0.81-1.04)$ and secondary prevention $(\mathrm{RR}=0.95,95 \% \mathrm{CI}=0.88-1.03)$. However, the CVD death reduction effect of statins was not observed for secondary prevention $(\mathrm{RR}=0.81,95 \% \mathrm{CI}=0.66-1.01)$ and mixed population $(R R=0.90,95 \% C I=0.73-1.12)$, but borderline for primary prevention $(R R=0.81$, 95\% CI $=0.66-1.01)$.

In the subgroup analysis by type of statins, risk reduction effects were only observed for pravastatin in terms of all-cause mortality and atorvastatin, pravastatin, fluvastatin, and pitavastatin in terms of CVD death. In contrast, the subgroup analysis by type of omega-3 fatty acids showed similar findings 
to those of the overall intervention. The pooled estimates and level of heterogeneity did not change much when excluding the REDUCE-IT trial, in which icosapentyl ethyl was used at a high dose of $4 \mathrm{~g}$.

Additional analyses by specific causes of death due to CVD found that statins were associated with lower risks of coronary heart disease death $(\mathrm{RR}=0.87,95 \% \mathrm{CI}=0.78-0.97)$ and fatal myocardial infarction $(\mathrm{RR}=0.73,95 \% \mathrm{CI}=0.57-0.93)$, but nonsignificant results for fatal stroke and heart failure death (Figure A10). In contrast, omega-3 supplementation was not associated with any specific causes of CVD death (Figure A11).

Dose-response meta-analysis for the effect of statins and omega-3 supplementation on all-cause mortality and CV death is summarized in Table A4 and Figures A12 and A13. There was a dose-response relationship between rosuvastatin and all-cause mortality, with a $9 \%$ decrement in all-cause mortality (per 10-mg RR =0.91, 95\% CI =0.80-0.99). There was also dose-response relationship between atorvastatin, pravastatin, simvastatin, fluvastatin, and omega-3 supplementation and CVD death, with per 10-mg RRs (95\% CI) of 0.90 (0.83-0.98), 0.96 (0.94-0.98), 0.82 (0.74-0.91), and 0.92 (0.86-0.98) for statins and per 1000-mg RR (95\% CI) of 0.94 (0.89-0.99) for omega-3 supplementation.

\subsection{Network Meta-Analysis}

An NMA combining direct and indirect estimates for pairwise comparisons among omega-3 supplementation, statins, and placebo is shown in Table 2. Similar to findings from the meta-analysis, omega-3 supplementation showed a lower risk of all-cause mortality than placebo, but not to a significant extent $(\mathrm{RR}=0.97,95 \% \mathrm{CI}=0.92-1.03)$, whereas randomization to omega-3 supplementation reduced CVD death by $9 \%$ compared to placebo $(\mathrm{RR}=0.91,95 \% \mathrm{CI}=0.84-0.99)$. Statins also demonstrated significant risk reductions for all-cause mortality and CVD death of 12\% and 16\%, with RRs (95\% CIs) of 0.88 (0.84-0.93) and 0.84 (0.78-0.81), respectively. A significant risk reduction was observed for the effects of statin use versus omega-3 supplementation on all-cause mortality $(\mathrm{RR}=0.91,95 \% \mathrm{CI}=0.85-0.98)$ but borderline on CVD death $(\mathrm{RR}=0.92,95 \% \mathrm{CI}=0.82-1.04)$.

Table 2. Relative risks and 95\% confidence intervals from network meta-analysis of omega-3 supplementation, statins, and placebo effects on all-cause mortality and cardiovascular disease death.

\begin{tabular}{ccc}
\hline Comparison & All-Cause Mortality & Cardiovascular Disease Death \\
\hline Overall & & \\
Statins vs. placebo & $\mathbf{0 . 8 8}(\mathbf{0 . 8 4 - 0 . 9 3 )}$ & $\mathbf{0 . 8 4}(\mathbf{0 . 7 8 - 0 . 9 1 )}$ \\
Omega-3 vs. placebo & $0.97(0.92-1.03)$ & $\mathbf{0 . 9 1}(\mathbf{0 . 8 4 - 0 . 9 9 )}$ \\
Statins vs. omega-3 & $\mathbf{0 . 9 1}(\mathbf{0 . 8 5 - 0 . 9 8 )}$ & $0.92(0.82-1.04)$ \\
Primary prevention & & \\
Statins vs. placebo & $0.89(0.78-1.01)$ & $\mathbf{0 . 7 7}(\mathbf{0 . 6 5 - 0 . 9 3 )}$ \\
Omega-3 vs. placebo & $0.97(0.91-1.03)$ & $0.91(0.81-1.03)$ \\
Statins vs. omega-3 & $0.92(0.80-1.06)$ & $0.85(0.68-1.05)$ \\
Secondary prevention & & $0.90(0.66-1.22)$ \\
Statins vs. placebo & $0.93(0.84-1.04)$ & $0.93(0.78-1.10)$ \\
Omega-3 vs. placebo & $0.95(0.87-1.04)$ & $0.97(0.66-1.43)$ \\
Statins vs. omega-3 & $0.98(0.85-1.13)$ & \\
Mixed population & & $\mathbf{0 . 8 4}(\mathbf{0 . 7 6 - 0 . 9 3 )}$ \\
Statins vs. placebo & $\mathbf{0 . 8 7}(\mathbf{0 . 8 1}-\mathbf{0 . 9 3 )}$ & $0.91(0.76-1.09)$ \\
Omega-3 vs. placebo & $0.98(0.88-1.10)$ & $0.92(0.75-1.14)$ \\
Statins vs. omega-3 & $0.88(0.77-1.01)$ &
\end{tabular}

Bold font indicates statistical significance.

In the subgroup analysis of primary prevention, there were borderline effects of statin use and omega-3 supplementation on all-cause mortality $(\mathrm{RR}=0.92,95 \% \mathrm{CI}=0.80-1.06)$ and CVD death $(\mathrm{RR}=0.85,95 \% \mathrm{CI}=0.68-1.05)$. However, nonsignificant differences were observed for both all-cause mortality and CVD death in the subgroup analysis of secondary prevention, with RRs (95\% CIs) of $0.98(0.85-1.13)$ and $0.97(0.66-1.43)$, respectively. In the subgroup analysis of mixed population, 
statin use was found to show borderline effect on all-cause mortality in the comparison with omega-3 supplementation $(\mathrm{RR}=0.88,95 \% \mathrm{CI}=0.77-1.01)$, whereas the effect on CVD death was not significantly different $(\mathrm{RR}=0.92,95 \% \mathrm{CI}=0.75-1.14)$.

\section{Discussion}

We performed a systematic review, meta-analysis, and NMA to summarize the current evidence on the effects of statin use and omega-3 supplementation on mortality outcomes. In this study, analyse of statins showed a statistically significant $10 \%$ reduction in the risk of all-cause mortality and a $14 \%$ reduction in the risk of CVD death. Additionally, the meta-analysis showed that omega-3 supplementation did not lead to a significantly lower risk of all-cause mortality, whereas the risk of CVD death was reduced significantly by $8 \%$. In the comparison with omega-3 supplementation, statins were found to significantly reduce all-cause mortality in the total population, borderline in the subgroup of primary prevention and mixed population, but not in the subgroup of secondary prevention. Furthermore, the NMA showed that the effects of statins and omega-3 supplementation on CVD death were borderline in the total population and in the subgroup of primary prevention, but not in the subgroups of secondary prevention and mixed population.

Our findings for the effect of statins are consistent with the latest updated meta-analysis, in which $11 \%$ and $20 \%$ risk reductions for all-cause mortality (24 RCTs) and CVD death (15 RCTs) were observed with the intervention of statins [8]. More RCTs were included in the current meta-analysis, with a similar effect on all-cause mortality (29 RCTs, $10 \%$ versus $11 \%$ risk reduction) but a smaller effect on CVD death (26 RCTs, $14 \%$ versus $20 \%$ risk reduction), although no statistical test was performed to compare the difference. Statins can change plasma levels of total cholesterol (TC), triglyceride (TG), high-density lipoprotein (HDL-C), and low-density lipoprotein (LDL-C) by inhibiting the synthesis of cholesterol in the liver by 3-hydroxy-3-methylglutaryl-CoA (HMG-CoA) reductase [77-83], thereby reducing the risk of CVD. However, most of the individual RCTs did not detect a significantly beneficial effect of statins on mortality outcomes. Randomization to statins might have led to a decrease in both all-cause mortality and CVD death in the LIPID and 4S trials [48,84]. More additional RCTs observed a significant effect of statins on all-cause mortality than on CVD death $[26,37,41,60]$, but the effect was closer to the null. Since more RCTs found a significant effect, substantial heterogeneity remained among RCTs investigating the association between statin use and all-cause mortality $\left(\mathrm{I}^{2}=43.8 \%\right.$, $p=0.006)$. Furthermore, recent studies found that statin use was associated with a lower risk of mortality in patients receiving clopidogrel $(\mathrm{RR}=0.54,95 \% \mathrm{CI}=0.40-0.74)$ [85] and Asian patients with type 2 diabetes [86]. Findings from another prospective cohort study of nearly 86,000 participants also supported the beneficial effect of statins on all-cause mortality (hazard ratio $0.86,95 \% \mathrm{CI}=0.77-0.95$ ) and CVD death (hazard ratio $0.75,95 \% \mathrm{CI}=0.64-0.89$ ) [87]. Moreover, our additional analyses by specific causes of death by CVD suggested the potential effect of statins on risk reductions of coronary heart disease death and fatal myocardial infarction.

The findings are in line with a recent meta-analysis by Abdelhamid et al., who reported that higher intake of omega-3 fatty acids had little or no effect on all-cause mortality and CVD death [13]. Apart from a different methodological approach, we included more up-to-date RCTs in this study, but excluded several RCTs with issues that might cause discrepancies. Several lipid biomarkers, including TC, TG, HDL-C, and LDL-C have been reported to be associated with CVD risk [88-91]. Omega-3 fatty acids can decrease plasma TG levels by reducing the production of hepatic very-low-density lipoprotein cholesterol and increasing chylomicron clearance. Furthermore, omega-3 fatty acids can change dysfunctional HDL-C to functional HDL-C and also exert weak TC- and LDL-C lowering effects [92-94]. In contrast with the findings of the pooled analysis, most of the individual RCTs did not find that omega-3 supplementation led to a significant reduction of mortality outcomes. The results of the GISSI-P trial stand apart from the null findings of 19 RCTs that examined the association between omega-3 supplementation and all-cause mortality, with an RR of 0.87 (95\% CI = 0.77-0.97) (Figures A1 and A3) [40]. Therefore, the pooled effect size for the effect of omega-3 supplementation on all-cause 
mortality was non-significantly different, as expected. In terms of CVD death, the results from the REDUCE-IT [40], ASCEND [24], and GISSI-P [40] trials, which accounted for $31.4 \%$ of the sample size, were only borderline significant, with RRs and 95\% CIs of 0.82 (0.67-0.99), 0.82 (0.67-0.99), and 0.84 (0.72-0.97), respectively (Figures A2 and A4). However, the pooled analysis with a large sample showed that omega-3 supplementation had a significant effect on CVD death, with low heterogeneity $\left(\mathrm{I}^{2}=6.0 \%, p=0.38\right)$. Furthermore, subgroup analyses by specific causes of death due to CVD showed no significant associations between omega-3 supplementation and the deaths of coronary heart disease, myocardial infarction, stroke, and heart failure.

In this study, we found a significantly lower risk of all-cause mortality in the statin group than the omega-3 supplementation group in primary prevention but not in secondary prevention. Because we defined studies of primary prevention as those with high probabilities of CVD risk factors, statin use was therefore hypothesized to reduce the death of CVD risk factor-related diseases. Moreover, statins were observed for their beneficial effects on reducing the mortality from breast, colorectal, kidney, ovarian, and prostate cancers, which contributed to all-cause mortality [95]. However, those effects of omega-3 supplementation have not been adequately investigated. In contrast, the effect of statins and omega-3 supplementation on CVD death reduction was borderline only. The mechanism is unclear, but a possible explanation may be found in the overlap of the pleiotropic effects of statins with the actions of omega-3 supplementation with, including endothelial function improvement, anti-thrombotic effects, and antioxidant effects [96]. Although previous RCTs and meta-analysis examined the effect of combination therapy with statins and omega-3 supplementation versus statins alone in patients with dyslipidemia [97-99] or cardiovascular events [92], the effects of statins and omega-3 supplementation have not been investigated. Our methodology was designed to minimize variety in the placebo group, which is the mediating factor in indirect comparisons between statins and omega-3 supplementation. We excluded RCTs if the placebo group contained omega- 6 or even low doses of omega- 3 to avoid bias in our pooled estimates. Furthermore, a subgroup analysis by primary and secondary prevention was performed to obtain robust findings.

Despite its strengths, there are certain limitations of our study. First, considering the nature of the study, potential heterogeneity in the associations with CVD death remained. Second, due to the lack of data, we were unable to perform the sex-specific meta-analysis to test whether there were different effects in males and females. Lastly, although both EPA alone [75] and the highly purified and stable EPA ethyl ester [28] have been determined to have beneficial effects, a pooled analysis with the other form of EPA + DHA might have introduced heterogeneity due to diversity. Combinations of different statin types might have also led to heterogeneity. Nevertheless, this is an up-to-date study with 278,954 participants that compared the effects of statin use and omega-3 supplementation on mortality outcomes.

\section{Conclusions}

In summary, statin use was significantly associated with decreased risks of mortality outcomes, whereas omega-3 supplementation showed nonsignificant or little effect on all-cause mortality and CVD death. Statin use was shown to be more effective in reducing all-cause mortality than omega-3 supplementation. However, the effect was borderline in terms of CVD mortality. Future direct comparisons between omega- 3 supplementation and statin use are required to detect the statistical benefits of omega-3 supplementation.

Author Contributions: Conceptualization, J.K., T.H., J.-M.K., S.Y.B., J.-H.C., E.P. (Eunmi Park), S.-M.L., E.P. (Eunju Park), J.Y.M., I.S.L., S.Y.Y., and J.-Y.Y.; methodology, J.K., T.H., J.-M.K., S.Y.B., J.-H.C., E.P. (Eunju Park), S.-M.L., E.P. (Eunmi Park), J.Y.M., I.S.L., S.Y.Y., and J.-Y.Y.; validation, J.K., J.-M.K., S.Y.B., and J.-H.C.; formal analysis, T.H.; data curation, J.K. and T.H.; writing-original draft preparation, T.H., and J.K.; and writing-review and editing, J.K., T.H., J.-M.K., S.Y.B., J.-H.C., E.P. (Eunju Park), S.-M.L., E.P. (Eunmi Park), J.Y.M., I.S.L., S.Y.Y., and J.-Y.Y. All authors have read and agreed to the published version of the manuscript.

Funding: This study was supported by the Korean Society of Lipid and Atherosclerosis and National Cancer Center, Korea (1910330). The funders had no role in the design, analysis, and interpretation of data.

Conflicts of Interest: The authors declare no conflict of interest. 


\section{Appendix A}

Table A1. General characteristics of the included randomized controlled trials.

\begin{tabular}{|c|c|c|c|c|c|c|c|}
\hline Study & Countries & Period & Sample Size & Age (Years) & Male & $\begin{array}{l}\text { Follow-Up } \\
\text { (Years) }\end{array}$ & $\begin{array}{c}\text { Body Mass Index } \\
\left(\mathrm{kg} / \mathrm{m}^{2}\right)\end{array}$ \\
\hline \multicolumn{8}{|l|}{ Statins } \\
\hline \multicolumn{8}{|l|}{ Primary prevention } \\
\hline Bestehorn 1997 (CIS) & Germany & 1989-1992 & 254 & 49.8 & $254(100 \%)$ & 2.3 & \\
\hline Blankenhorn 1993 (MARS) & & 1985-1989 & 247 & 58 & $225(91 \%)$ & 2.2 & \\
\hline Colhoun 2004 (CARDS) & United Kingdom and Ireland & 1997-2001 & 2838 & 62 & $1930(68 \%)$ & 3.9 & \\
\hline Knopp 2006 (ASPEN) & 14 countries & 1996-1999 & 2410 & 61 & $1598(66.3 \%)$ & 4 & 28.9 \\
\hline Sever 2004 (ASCOT-LLA) & United Kingdom and Ireland & 1998-2000 & 10305 & 63 & $8347(81 \%)$ & 5 & 28.6 \\
\hline Teo 2000 (SCAT) & Canada & 1996-1998 & 460 & 61 & $409(89 \%)$ & 4 & \\
\hline Wanner 2005 & Germany & 1998-2002 & 1255 & 65.7 & $676(53.9 \%)$ & 4 & 27.5 \\
\hline \multicolumn{8}{|l|}{ Secondary prevention } \\
\hline Kjekshus 2007 (CORONA) & 21 countries & 2003-2005 & 5011 & 73 & $3808(76 \%)$ & 2.7 & 27 \\
\hline Koren 2004 (ALLIANCE) & United States & 1995-2002 & 2442 & 61.2 & $2007(82.2 \%)$ & 4.5 & \\
\hline Liem 2002 (FLORIDA) & Netherlands & 1997-1999 & 540 & 60.5 & $448(83 \%)$ & 1 & \\
\hline \multicolumn{8}{|l|}{ Mixed population } \\
\hline Amarenco 2006 (SPARCL) & Multi-centers & 1998-2001 & 4731 & 62.7 & $2824(59.7 \%)$ & 4.9 & 27.4 \\
\hline Asselbergs 2004 (PREVEND IT) & & & 864 & 51.3 & $562(65 \%)$ & 3.8 & 24.1 \\
\hline Blankenhorn 1993 (MARS) & & 1985-1989 & 247 & 58 & $225(91 \%)$ & 2.2 & 26 \\
\hline Chan 2010 (ASTRONOMER) & Canada & 2002-2005 & 269 & 58 & $166(61.7 \%)$ & 3.5 & 28.1 \\
\hline Davis 2002 (ALLHAT-LTT) & North America & 1994-2002 & 10355 & 66.4 & $5312(51.3 \%)$ & 4.8 & 29.9 \\
\hline Downs 1998 (AFCAPS/TexCAPS) & United States & 1990-1993 & 6605 & 58 & $5608(84.9 \%)$ & 5.2 & 25 \\
\hline Emberson 2011 (MRC/BHF) & United Kingdom & 1994-1997 & 20536 & 64 & $16244(79.1 \%)$ & 5 & 27.6 \\
\hline Fellstrom 2009 (AURORA) & 25 countries & 2003-2004 & 2773 & 64.2 & $1722(62.1 \%)$ & 3.8 & 25.4 \\
\hline Ford 2016 (WOSCOPS) & Scotland & 1989-1991 & 6595 & 55 & $6595(100 \%)$ & 4.9 & \\
\hline Furberg 1994 (ACAPS) & United States & 1989-1990 & 919 & 62 & $478(52 \%)$ & 2.8 & 25.9 \\
\hline Furberg 1995 (PLAC-I and -II) & & & 559 & 58 & $159(28.4 \%)$ & 3 & \\
\hline Makuuchi 2005 (PCABG) & Japan & 1991-1994 & 303 & 58.9 & $255(84.2 \%)$ & 4.5 & 23.7 \\
\hline Nakagawa 2004 (PCS) & Japan & 1991-1995 & 120 & 55 & $109(91.2 \%)$ & 5.4 & \\
\hline Nakamura 2006 (MEGA) & Japan & 1994-1999 & 7832 & 58.3 & $2428(31 \%)$ & 5.3 & 23.8 \\
\hline Ostadal 2010 (FACS) & Czech Republic & $2003-2006$ & 156 & 62.1 & $106(68 \%)$ & 1 & \\
\hline
\end{tabular}


Table A1. Cont.

\begin{tabular}{|c|c|c|c|c|c|c|c|}
\hline Study & Countries & Period & Sample Size & Age (Years) & Male & $\begin{array}{l}\text { Follow-Up } \\
\text { (Years) }\end{array}$ & $\begin{array}{c}\text { Body Mass Index } \\
\left(\mathrm{kg} / \mathrm{m}^{2}\right)\end{array}$ \\
\hline Pedersen 1994 (4S) & Scandinavia & $1988-1989$ & 4444 & 58.6 & $3617(81.4 \%)$ & 5.4 & 26 \\
\hline Ridker 2008 (JUPITER) & 26 countries & 2003-2006 & 17802 & 66 & $11002(61.8 \%)$ & 1.9 & 28.4 \\
\hline Riegger 1999 & Germany and Czech Republic & & 365 & 59.8 & $225(61.6 \%)$ & 1 & \\
\hline Sawayama 2002 (FAST) & Japan & 1996-2000 & 164 & 66.3 & $52(31.7 \%)$ & 2 & 23.2 \\
\hline Serruys 2002 (LIPS) & Europe, Canada, and Brazil & $1996-1998$ & 1677 & 60 & $1405(83.8 \%)$ & 3.9 & 26.6 \\
\hline Shepherd 2002 (PROSPER) & Ireland and Netherlands & 1997-1999 & 5804 & 75.3 & $3825(65.9 \%)$ & 3.2 & 26.8 \\
\hline Takano 2013 (PEARL) & Japan & $2006-2008$ & 574 & 62.6 & $468(81.5 \%)$ & 3 & \\
\hline Tavazzi 2008 (GISSI-HF) & Italy & $2002-2005$ & 4574 & 68 & $3540(77.4 \%)$ & 3.9 & 27.1 \\
\hline Tonkin 1998 (LIPID) & Australia and New Zealand & 1990-1992 & 9014 & 62 & $7482(83 \%)$ & 6.1 & \\
\hline Yokoi 2005 (ATHEROMA) & Japan & 1994-1997 & 288 & 59.3 & $239(83 \%)$ & 3 & \\
\hline Yusuf 2016 (HOPE-3) & 21 countries & $2007-2010$ & 12705 & 63.8 & $6835(53.8 \%)$ & 5.6 & 27.1 \\
\hline \multicolumn{8}{|l|}{$\begin{array}{l}\text { Omega-3 supplementation } \\
\text { Primary prevention }\end{array}$} \\
\hline Andrieu 2017 (MAPT) & France and Monaco & 2008-2011 & 1652 & 75.3 & $595(36 \%)$ & 3 & 26.1 \\
\hline Bhatt 2019 (REDUCE_IT) & 11 countries & 2011-2016 & 8159 & 64 & $5809(71.2 \%)$ & 4.9 & \\
\hline Bowman 2018 (ASCEND) & United Kingdom & 2005-2011 & 15480 & 63.3 & $9690(62.6 \%)$ & 7.4 & 29.8 \\
\hline Roncaglioni 2013 (Risk and Prevention) & Italy & $2004-2007$ & 12505 & 64 & $7691(61.5 \%)$ & 5 & 30.8 \\
\hline Bosch 2012 (ORIGIN) & 40 countries & 2003-2005 & 12536 & 63.5 & $8148(65 \%)$ & 6.2 & \\
\hline \multicolumn{8}{|l|}{ Secondary prevention } \\
\hline Kromhout 2010 (AlphaOmega) & Netherlands & 2002-2006 & 4837 & 69 & $3783(78.2 \%)$ & 3.4 & 27.8 \\
\hline Tavazzi 2008 (GISSI-HF) & Italy & $2002-2005$ & 6975 & 68 & $5454(78.2 \%)$ & 3.9 & 27 \\
\hline \multicolumn{8}{|l|}{ Mixed population } \\
\hline Bonds 2014 (AREDS2) & United States & 2006-2008 & 4203 & 74 & $1816(43.2 \%)$ & 4.8 & \\
\hline Brouwer 2006 (SOFA) & European countries & 2001-2004 & 546 & 61.5 & $459(84.1 \%)$ & 1 & 26.9 \\
\hline Dangour 2010 (OPAL) & England and Wales & $2005-2006$ & 867 & 75 & $477(55 \%)$ & 2 & 27.1 \\
\hline Einvik 2010 (DO IT) & Norwegian & & 563 & 70 & $563(100 \%)$ & 3 & \\
\hline Galan 2010 (SU.FOL.OM3) & France & 2003-2007 & 2501 & 61.4 & $1986(79.4 \%)$ & 4.7 & 27.2 \\
\hline Macchia 2013 (FORWARD) & & $2008-2011$ & 586 & 66.1 & $321(54.8 \%)$ & 1 & \\
\hline Manson 2019 (VITAL) & United States & 2010-2018 & 25871 & 67.1 & $12780(49.4 \%)$ & 5.3 & \\
\hline Nilsen 2001 & Norway & 1995-1996 & 300 & 64 & $238(79.3 \%)$ & 1.5 & 26 \\
\hline Raitt 2005 & United States & 1999-2003 & 200 & 62.5 & $172(86 \%)$ & 2 & \\
\hline Rauch 2009 (OMEGA) & German & 2003-2007 & 3804 & 64 & $2830(74.4 \%)$ & 1 & 27.5 \\
\hline Valagussa 1999 (GISSI-P) & Italy & 1993-1995 & 11334 & 59.4 & $9668(85.3 \%)$ & 3.5 & 26.5 \\
\hline Yokoyama 2007 (JELIS) & Japan & 1996-1999 & 18645 & 61 & $5855(31.4 \%)$ & 4.6 & 24 \\
\hline
\end{tabular}


Table A2. History of cardiovascular events and risk factors of cardiovascular disease at baseline.

\begin{tabular}{|c|c|c|c|c|c|c|c|c|c|}
\hline Study & $\begin{array}{l}\text { Cardiovascular } \\
\text { Disease }\end{array}$ & $\begin{array}{c}\text { Coronary } \\
\text { Heart Disease }\end{array}$ & $\begin{array}{l}\text { Myocardial } \\
\text { Infarction }\end{array}$ & Heart Failure & Hypertension & Dyslipidemia & Diabetes & Smoking & Obesity \\
\hline \multicolumn{10}{|l|}{ Statins } \\
\hline \multicolumn{10}{|l|}{ Primary prevention } \\
\hline Bestehorn 1997 (CIS) & & & & & & & & $214(84.3 \%)$ & \\
\hline Blankenhorn 1993 (MARS) & & & $148(60 \%)$ & & $114(46 \%)$ & & & $198(80 \%)$ & \\
\hline Colhoun 2004 (CARDS) & & & & & & & $2838(100 \%)$ & $1853(65.3 \%)$ & $1053(37.1 \%)$ \\
\hline Knopp 2006 (ASPEN) & & & $395(16.4 \%)$ & & $1328(55.1 \%)$ & $711(29.5 \%)$ & $2410(100 \%)$ & $299(12.4 \%)$ & \\
\hline Sever 2004 (ASCOT-LLA) & & & & & $10305(100 \%)$ & & $2535(24.6 \%)$ & $3370(32.7 \%)$ & \\
\hline Teo 2000 (SCAT) & & & $322(70 \%)$ & & $166(36 \%)$ & & $51(11 \%)$ & $377(82 \%)$ & \\
\hline Wanner 2005 & & & $221(17.6 \%)$ & $444(35.4 \%)$ & & & $1255(100 \%)$ & $507(40.4 \%)$ & \\
\hline \multicolumn{10}{|l|}{ Secondary prevention } \\
\hline Kjekshus 2007 (CORONA) & & & $3007(60 \%)$ & $5011(100 \%)$ & $3157(63 \%)$ & & $1478(29.5 \%)$ & $431(8.6 \%)$ & \\
\hline Koren 2004 (ALLIANCE) & & $2442(100 \%)$ & $1411(57.8 \%)$ & $161(6.6 \%)$ & & & $540(22.1 \%)$ & $476(19.5 \%)$ & \\
\hline Liem 2002 (FLORIDA) & & & $540(100 \%)$ & & & & & & \\
\hline \multicolumn{10}{|l|}{ Mixed population } \\
\hline Amarenco 2006 (SPARCL) & & & & & & & & $2791(59 \%)$ & \\
\hline Arthros 2002 (GREACE) & & & $1299(81.2 \%)$ & & $686(42.9 \%)$ & & $314(19.6 \%)$ & & \\
\hline Asselbergs 2004 (PREVEND IT) & $29(3.4 \%)$ & & $3(0.4 \%)$ & $0(0 \%)$ & & & $22(2.6 \%)$ & $629(72.8 \%)$ & \\
\hline Chan 2010 (ASTRONOMER) & & & & & & & & $130(48.3 \%)$ & \\
\hline Davis 2002 (ALLHAT-LTT) & & $1470(14.2 \%)$ & & & & & $3635(35.1 \%)$ & $2402(23.2 \%)$ & $4401(42.5 \%)$ \\
\hline Downs 1998 (AFCAPS/TexCAPS) & & & & & $1446(21.9 \%)$ & & $152(2.3 \%)$ & $819(12.4 \%)$ & \\
\hline Emberson 2011 (MRC/BHF) & & $13677(66.6 \%)$ & $8933(43.5 \%)$ & & & & $6243(30.4 \%)$ & $15361(74.8 \%)$ & \\
\hline Fellstrom 2009 (AURORA) & $1104(39.8 \%)$ & & $283(10.2 \%)$ & & & & $732(26.4 \%)$ & $430(15.5 \%)$ & \\
\hline Ford 2016 (WOSCOPS) & & & $0(0 \%)$ & & & & & $2902(44 \%)$ & \\
\hline Furberg 1994 (ACAPS) & & & & & $265(28.8 \%)$ & & & $519(56.5 \%)$ & \\
\hline Furberg 1995 (PLAC-I and -II) & & & $271(48.5 \%)$ & & $226(40.5 \%)$ & & $4(0.7 \%)$ & $87(15.5 \%)$ & \\
\hline Makuuchi 2005 (PCABG) & & & $188(62 \%)$ & & $156(51.5 \%)$ & & $101(33.3 \%)$ & $127(41.9 \%)$ & \\
\hline Nakagawa 2004 (PCS) & & & & & $71(59.2 \%)$ & $0(0 \%)$ & $21(17.5 \%)$ & $81(67.5 \%)$ & \\
\hline Nakamura 2006 (MEGA) & & $0(0 \%)$ & & & $3274(41.8 \%)$ & & $1629(20.8 \%)$ & $1613(20.6 \%)$ & \\
\hline Ostadal 2010 (FACS) & & & $12(7.7 \%)$ & & $80(51.3 \%)$ & $18(11.5 \%)$ & $30(19.2 \%)$ & $46(29.2 \%)$ & \\
\hline Pedersen 1994 (4S) & & & & & $1155(26 \%)$ & & $200(4.5 \%)$ & $3324(74.8 \%)$ & \\
\hline Ridker 2008 (JUPITER) & $0(0 \%)$ & $0(0 \%)$ & $0(0 \%)$ & $0(0 \%)$ & & & & $2813(15.8 \%)$ & \\
\hline
\end{tabular}


Table A2. Cont.

\begin{tabular}{|c|c|c|c|c|c|c|c|c|c|}
\hline Study & $\begin{array}{l}\text { Cardiovascular } \\
\text { Disease }\end{array}$ & $\begin{array}{c}\text { Coronary } \\
\text { Heart Disease }\end{array}$ & $\begin{array}{l}\text { Myocardial } \\
\text { Infarction }\end{array}$ & Heart Failure & Hypertension & Dyslipidemia & Diabetes & Smoking & Obesity \\
\hline Riegger 1999 & & & $130(35.6 \%)$ & & $107(29.3 \%)$ & $365(100 \%)$ & $20(5.5 \%)$ & $35(9.6 \%)$ & \\
\hline Sawayama 2002 (FAST) & & $68(41.5 \%)$ & & & $65(39.6 \%)$ & & $41(25 \%)$ & $95(57.8 \%)$ & \\
\hline Serruys 2002 (LIPS) & & & $745(44.4 \%)$ & & $647(38.6 \%)$ & & $201(12 \%)$ & $1199(71.5 \%)$ & \\
\hline Shepherd 2002 (PROSPER) & $2565(44.2 \%)$ & & $778(13.4 \%)$ & & $3593(61.9 \%)$ & & $621(10.7 \%)$ & $1555(26.8 \%)$ & \\
\hline Takano 2013 (PEARL) & & & $144(25.1 \%)$ & & $260(45.3 \%)$ & & $157(27.4 \%)$ & & \\
\hline Tavazzi 2008 (GISSI-HF) & & & & & $2484(54.3 \%)$ & & & $645(14.1 \%)$ & \\
\hline Tonkin 1998 (LIPID) & $1604(17.8 \%)$ & & & & $3759(41.7 \%)$ & & $784(8.7 \%)$ & $6607(73.3 \%)$ & $1614(17.9 \%)$ \\
\hline Yokoi 2005 (ATHEROMA) & & & $131(45.5 \%)$ & & $121(42 \%)$ & & $54(18.8 \%)$ & & \\
\hline Yusuf 2016 (HOPE-3) & $0(0 \%)$ & $0(0 \%)$ & $0(0 \%)$ & $0(0 \%)$ & $4815(37.9 \%)$ & $4587(36.1 \%)$ & $737(5.8 \%)$ & $3519(27.7 \%)$ & \\
\hline Omega-3 supplementation & \multicolumn{9}{|c|}{$\begin{array}{c}\text { Primary prevention } \\
\text { Andrieu } 2017 \text { (MAPT) }\end{array}$} \\
\hline Bhatt 2019 (REDUCE_IT) & & & & & & $8159(100 \%)$ & $4781(58.6 \%)$ & & $4683(57.4 \%)$ \\
\hline Bosch 2012 (ORIGIN) & $7371(58.8 \%)$ & & & & 9966 (79.5\%) & & & $1554(12.4 \%)$ & \\
\hline Bowman 2018 (ASCEND) & $0(0 \%)$ & $0(0 \%)$ & $0(0 \%)$ & $0(0 \%)$ & $7647(49.4 \%)$ & & $15480(100 \%)$ & $8328(53.8 \%)$ & $7198(46.5 \%)$ \\
\hline $\begin{array}{c}\text { Roncaglioni } 2013 \text { (Risk and } \\
\text { Prevention) }\end{array}$ & & & & $400(3.2 \%)$ & $10579(84.6 \%)$ & $8904(71.2 \%)$ & $7490(59.9 \%)$ & $2714(21.7 \%)$ & $6077(48.6 \%)$ \\
\hline \multicolumn{10}{|l|}{ Secondary prevention } \\
\hline Kromhout 2010 (AlphaOmega) & & & $4837(100 \%)$ & & 4339 (89.7\%) & $4160(86 \%)$ & $1016(21 \%)$ & $817(16.9 \%)$ & $1171(24.2 \%)$ \\
\hline Tavazzi 2008 (GISSI-HF) & & & $990(14.2 \%)$ & $6975(100 \%)$ & $3808(54.6 \%)$ & $1576(22.6 \%)$ & $1974(28.3 \%)$ & $990(14.2 \%)$ & \\
\hline \multicolumn{10}{|l|}{ Mixed population } \\
\hline Bonds 2014 (AREDS2) & $807(19.2 \%)$ & $147(3.5 \%)$ & $294(7 \%)$ & $147(3.5 \%)$ & $248(5.9 \%)$ & $1849(44 \%)$ & $546(13 \%)$ & $2379(56.6 \%)$ & \\
\hline Brouwer 2006 (SOFA) & & $384(70.3 \%)$ & $342(62.6 \%)$ & & $277(50.7 \%)$ & & $87(15.9 \%)$ & $366(67 \%)$ & \\
\hline Dangour 2010 (OPAL) & & & $34(3.9 \%)$ & & $485(55.9 \%)$ & & & & $201(23.2 \%)$ \\
\hline Einvik 2010 (DO IT) & $155(27.5 \%)$ & & & & $158(28 \%)$ & $110(19.5 \%)$ & $82(14.5 \%)$ & $191(34 \%)$ & \\
\hline Galan 2010 (SU.FOL.OM3) & & & $1150(46 \%)$ & & & & & $1821(72.8 \%)$ & \\
\hline Macchia 2013 (FORWARD) & & $69(11.7 \%)$ & & $83(14.1 \%)$ & $536(91.4 \%)$ & $274(46.7 \%)$ & $76(12.9 \%)$ & $247(42.2 \%)$ & \\
\hline Manson 2019 (VITAL) & & & & $0(0 \%)$ & $12884(49.8 \%)$ & $9702(37.5 \%)$ & $3544(13.7 \%)$ & $1863(7.2 \%)$ & \\
\hline Nilsen 2001 & & & $70(23.3 \%)$ & $26(8.7 \%)$ & $73(24.3 \%)$ & & $31(10.3 \%)$ & $227(75.7 \%)$ & \\
\hline Raitt 2005 & & $146(73 \%)$ & $111(55.5 \%)$ & & $101(50.5 \%)$ & $47(23.5 \%)$ & & & \\
\hline Rauch 2009 (OMEGA) & & & $548(14.4 \%)$ & & $2530(66.5 \%)$ & $1883(49.5 \%)$ & $1027(27 \%)$ & $1396(36.7 \%)$ & \\
\hline Valagussa 1999 (GISSI-P) & & & $1360(12 \%)$ & & $4035(35.6 \%)$ & & $1677(14.8 \%)$ & $8750(77.2 \%)$ & $1643(14.5 \%)$ \\
\hline Yokoyama 2007 (JELIS) & & & $1044(5.6 \%)$ & & $6619(35.5 \%)$ & & $3039(16.3 \%)$ & $3524(18.9 \%)$ & \\
\hline
\end{tabular}


Table A3. Treatment arms and outcome events of studies included in the final analysis.

\begin{tabular}{|c|c|c|c|c|c|c|c|c|}
\hline \multirow[b]{2}{*}{ Study } & \multicolumn{4}{|c|}{ Intervention Arm } & \multicolumn{4}{|c|}{ Comparison Arm } \\
\hline & Daily Dose & Sample Size & $\begin{array}{l}\text { All-Cause } \\
\text { Mortality }\end{array}$ & CVD Death & Daily Dose & Sample Size & $\begin{array}{l}\text { All-Cause } \\
\text { Mortality }\end{array}$ & CVD Death \\
\hline \multicolumn{9}{|l|}{ Statins } \\
\hline \multicolumn{9}{|l|}{ Primary prevention } \\
\hline Bestehorn 1997 (CIS) & Simvastatin $40 \mathrm{mg}$ & 129 & & $1(0.8 \%)$ & Placebo & 125 & & $2(1.6 \%)$ \\
\hline Blankenhorn 1993 (MARS) & Lovastatin $80 \mathrm{mg}$ & 123 & $1(0.8 \%)$ & & Placebo & 124 & $2(1.6 \%)$ & \\
\hline Colhoun 2004 (CARDS) & Atorvastatin $10 \mathrm{mg}$ & 1428 & $61(4.3 \%)$ & $134(9.4 \%)$ & Placebo & 1410 & $82(5.8 \%)$ & $189(13.4 \%)$ \\
\hline Knopp 2006 (ASPEN) & Atorvastatin $10 \mathrm{mg}$ & 1211 & $70(5.8 \%)$ & & Placebo & 1199 & $68(5.7 \%)$ & \\
\hline Sever 2004 (ASCOT-LLA) & Atorvastatin $10 \mathrm{mg}$ & 5168 & $185(3.6 \%)$ & $74(1.4 \%)$ & Placebo & 5137 & $212(4.1 \%)$ & $82(1.6 \%)$ \\
\hline Teo 2000 (SCAT) & Simvastatin $10 \mathrm{mg}$ & 230 & $13(5.7 \%)$ & $7(3 \%)$ & Placebo & 230 & $6(2.6 \%)$ & $4(1.7 \%)$ \\
\hline Wanner 2005 & Atorvastatin $20 \mathrm{mg}$ & 619 & $297(48 \%)$ & $121(19.5 \%)$ & Placebo & 636 & $320(50.3 \%)$ & $149(23.4 \%)$ \\
\hline \multicolumn{9}{|l|}{ Secondary prevention } \\
\hline Kjekshus 2007 (CORONA) & Rosuvastatin $10 \mathrm{mg}$ & 2514 & $728(29 \%)$ & $581(23.1 \%)$ & Placebo & 2497 & $759(30.4 \%)$ & $593(23.7 \%)$ \\
\hline Koren 2004 (ALLIANCE) & Atorvastatin up to $80 \mathrm{mg}$ & 1217 & $121(9.9 \%)$ & $43(3.5 \%)$ & Usual care & 1225 & $127(10.4 \%)$ & $61(5 \%)$ \\
\hline Liem 2002 (FLORIDA) & Fluvastatin $80 \mathrm{mg}$ & 265 & $7(2.6 \%)$ & $6(2.3 \%)$ & Placebo & 275 & $11(4 \%)$ & $11(4 \%)$ \\
\hline \multicolumn{9}{|l|}{ Mixed population } \\
\hline Amarenco 2006 (SPARCL) & Atorvastatin $80 \mathrm{mg}$ & 2365 & $216(9.1 \%)$ & $78(3.3 \%)$ & Placebo & 2366 & $211(8.9 \%)$ & $98(4.1 \%)$ \\
\hline Arthros 2002 (GREACE) & Atorvastatin $10-80 \mathrm{mg}$ & 800 & $23(2.9 \%)$ & & Usual care & 800 & $40(5 \%)$ & \\
\hline Asselbergs 2004 (PREVEND IT) & Pravastatin $40 \mathrm{mg}$ & 433 & & $4(0.9 \%)$ & Placebo & 431 & & $4(0.9 \%)$ \\
\hline Chan 2010 (ASTRONOMER) & Rosuvastatin $40 \mathrm{mg}$ & 134 & & $2(1.5 \%)$ & Placebo & 135 & & $5(3.7 \%)$ \\
\hline Davis 2002 (ALLHAT-LTT) & Pravastatin $40 \mathrm{mg}$ & 5170 & $631(12.2 \%)$ & $295(5.7 \%)$ & Usual care & 5185 & $641(12.4 \%)$ & $300(5.8 \%)$ \\
\hline Study & Daily Dose & Sample Size & $\begin{array}{l}\text { All-Cause } \\
\text { Mortality }\end{array}$ & CVD Death & Daily Dose & Sample Size & $\begin{array}{l}\text { All-Cause } \\
\text { Mortality }\end{array}$ & CVD Death \\
\hline Downs 1998 (AFCAPS/TexCAPS) & Lovastatin $20 \mathrm{mg}$ & 3304 & & $17(0.5 \%)$ & Placebo & 3301 & & $25(0.8 \%)$ \\
\hline Emberson 2011 (MRC/BHF) & Simvastatin $40 \mathrm{mg}$ & 10269 & $1328(12.9 \%)$ & & Placebo & 10267 & $1507(14.7 \%)$ & \\
\hline Fellstrom 2009 (AURORA) & Rosuvastatin $10 \mathrm{mg}$ & 1389 & $636(45.8 \%)$ & $324(23.3 \%)$ & Placebo & 1384 & $660(47.7 \%)$ & $324(23.4 \%)$ \\
\hline Ford 2016 (WOSCOPS) & Pravastatin $40 \mathrm{mg}$ & 3302 & $619(18.7 \%)$ & $252(7.6 \%)$ & Placebo & 3293 & $674(20.5 \%)$ & $297(9 \%)$ \\
\hline Furberg 1994 (ACAPS) & $\begin{array}{l}\text { Lovastatin } 20-40 \mathrm{mg}( \pm \\
\text { warfarin } 1 \mathrm{mg})\end{array}$ & 460 & $1(0.2 \%)$ & & $\begin{array}{c}\text { Placebo }( \pm \\
\text { warfarin } 1 \mathrm{mg})\end{array}$ & 459 & $8(1.7 \%)$ & \\
\hline Furberg 1995 (PLAC-I and -II) & Pravastatin 20 or $40 \mathrm{mg}$ & 281 & $7(2.5 \%)$ & $5(1.8 \%)$ & Placebo & 278 & $11(4 \%)$ & $5(1.8 \%)$ \\
\hline Makuuchi 2005 (PCABG) & Pravastatin 10-20 mg & 152 & $6(3.9 \%)$ & $4(2.6 \%)$ & $\begin{array}{c}\text { No lipid } \\
\text { lowering agent }\end{array}$ & 151 & $11(7.3 \%)$ & $4(2.6 \%)$ \\
\hline Nakagawa 2004 (PCS) & Pravastatin $10 \mathrm{mg}$ & 54 & $17(31.5 \%)$ & $2(3.7 \%)$ & Dietary control & 66 & $23(34.8 \%)$ & $1(1.5 \%)$ \\
\hline Nakamura 2006 (MEGA) & Pravastatin $10-20 \mathrm{mg}$ & 3866 & $55(1.4 \%)$ & $11(0.3 \%)$ & Dietary control & 3966 & $79(2 \%)$ & $18(0.5 \%)$ \\
\hline Ostadal 2010 (FACS) & Fluvastatin $80 \mathrm{mg}$ & 78 & & $1(1.3 \%)$ & Placebo & 78 & & $4(5.1 \%)$ \\
\hline Pedersen 1994 (4S) & Simvastatin $20 \mathrm{mg}$ & 2221 & $182(8.2 \%)$ & $136(6.1 \%)$ & Placebo & 2223 & $256(11.5 \%)$ & $207(9.3 \%)$ \\
\hline Ridker 2008 (JUPITER) & Rosuvastatin $20 \mathrm{mg}$ & 8901 & $198(2.2 \%)$ & & Placebo & 8901 & $247(2.8 \%)$ & \\
\hline Riegger 1999 & Fluvastatin 40 or $80 \mathrm{mg}$ & 187 & & $2(1.1 \%)$ & Placebo & 178 & & $4(2.2 \%)$ \\
\hline Sawayama 2002 (FAST) & Pravastatin $10 \mathrm{mg}$ & 83 & $5(6 \%)$ & & Dietary control & 81 & $9(11.1 \%)$ & \\
\hline
\end{tabular}


Table A3. Cont.

\begin{tabular}{|c|c|c|c|c|c|c|c|c|}
\hline \multirow[b]{2}{*}{ Serruys 2002 (LIPS) } & \multicolumn{4}{|c|}{ Intervention Arm } & \multicolumn{4}{|c|}{ Comparison Arm } \\
\hline & Fluvastatin $80 \mathrm{mg}$ & 844 & $36(4.3 \%)$ & $13(1.5 \%)$ & Placebo & 833 & $49(5.9 \%)$ & $24(2.9 \%)$ \\
\hline Shepherd 2002 (PROSPER) & Pravastatin $40 \mathrm{mg}$ & 2891 & $298(10.3 \%)$ & & Placebo & 2913 & $306(10.5 \%)$ & \\
\hline Takano 2013 (PEARL) & Pitavastatin $2 \mathrm{mg}$ & 288 & $27(9.4 \%)$ & $24(8.3 \%)$ & No statin & 286 & $37(12.9 \%)$ & $22(7.7 \%)$ \\
\hline Tavazzi 2008 (GISSI-HF) & Rosuvastatin $10 \mathrm{mg}$ & 2285 & $657(28.8 \%)$ & $478(20.9 \%)$ & Placebo & 2289 & $644(28.1 \%)$ & $488(21.3 \%)$ \\
\hline Tonkin 1998 (LIPID) & Pravastatin $40 \mathrm{mg}$ & 4512 & $498(11 \%)$ & $331(7.3 \%)$ & Placebo & 4502 & $633(14.1 \%)$ & $433(9.6 \%)$ \\
\hline Yokoi 2005 (ATHEROMA) & $\begin{array}{c}\text { Pravastatin } 10-20 \mathrm{mg}+ \\
\text { diet }\end{array}$ & 142 & $1(0.7 \%)$ & & Dietary control & 146 & $2(1.4 \%)$ & \\
\hline Yusuf 2016 (HOPE-3) & Rosuvastatin $10 \mathrm{mg}$ & 6361 & $334(5.3 \%)$ & $154(2.4 \%)$ & Placebo & 6344 & $357(5.6 \%)$ & $171(2.7 \%)$ \\
\hline \multicolumn{9}{|l|}{$\begin{array}{c}\text { Omega-3 supplementation } \\
\text { Primary prevention }\end{array}$} \\
\hline Andrieu 2017 (MAPT) & $\begin{array}{l}\text { EPA + DHA up to } \\
300 / 1300 \mathrm{mg}\end{array}$ & 820 & $20(2.4 \%)$ & & Placebo & 832 & $20(2.4 \%)$ & \\
\hline Bhatt 2019 (REDUCE_IT) & Icosapent ethyl $4 \mathrm{~g}$ & 4069 & $274(6.7 \%)$ & $174(4.3 \%)$ & Placebo & 4090 & $310(7.6 \%)$ & $213(5.2 \%)$ \\
\hline Bosch 2012 (ORIGIN) & EPA + DHA 465/375 mg & 6281 & $951(15.1 \%)$ & $574(9.1 \%)$ & Olive oil & 6255 & $964(15.4 \%)$ & $581(9.3 \%)$ \\
\hline Bowman 2018 (ASCEND) & EPA + DHA 460/380 mg & 7740 & $752(9.7 \%)$ & $186(2.4 \%)$ & Olive oil & 7740 & $788(10.2 \%)$ & $228(2.9 \%)$ \\
\hline $\begin{array}{c}\text { Roncaglioni } 2013 \text { (Risk and } \\
\text { Prevention) } \\
\text { Secondary prevention }\end{array}$ & $\begin{array}{c}\text { EPA + DHA } 1 \mathrm{~g} \text { (ratio } \\
0.9: 1 \text { to } 1.5: 1 \text { ) }\end{array}$ & 6239 & $348(5.6 \%)$ & $142(2.3 \%)$ & Olive oil & 6266 & $337(5.4 \%)$ & $137(2.2 \%)$ \\
\hline Kromhout 2010 (AlphaOmega) & $\begin{array}{c}\mathrm{EPA}+\mathrm{DHA} 400 \mathrm{mg}( \pm \\
\text { ALA } 2 \mathrm{~g})\end{array}$ & 2404 & $186(7.7 \%)$ & $80(3.3 \%)$ & $\begin{array}{l}\text { Control ( } \pm \text { ALA } \\
2 \mathrm{~g})\end{array}$ & 2433 & $184(7.6 \%)$ & $82(3.4 \%)$ \\
\hline Tavazzi 2008 (GISSI-HF) & $\begin{array}{c}\mathrm{EPA}+\mathrm{DHA} 850 \text { to } 882 \\
\text { mg }\end{array}$ & 3494 & $2157(61.7 \%)$ & $712(20.4 \%)$ & Placebo & 3481 & $2202(63.3 \%)$ & $765(22 \%)$ \\
\hline Mixed population & & & & & & & & \\
\hline Bonds 2014 (AREDS2) & EPA + DHA 650/350 mg & 2147 & $200(9.3 \%)$ & $14(0.7 \%)$ & Placebo & 2056 & $168(8.2 \%)$ & $13(0.6 \%)$ \\
\hline Brouwer 2006 (SOFA) & EPA + DHA 464/335 mg & 273 & $8(2.9 \%)$ & $6(2.2 \%)$ & Sunflower oil & 273 & $14(5.1 \%)$ & $13(4.8 \%)$ \\
\hline Einvik 2010 (DO IT) & EPA + DHA 1176/840 mg & 282 & $14(5 \%)$ & $7(2.5 \%)$ & Placebo & 281 & $24(8.5 \%)$ & $11(3.9 \%)$ \\
\hline Galan 2010 (SU.FOL.OM3) & $\mathrm{EPA}+\mathrm{DHA} 400 / 200 \mathrm{mg}$ & 1253 & $58(4.6 \%)$ & & Placebo & 1248 & $59(4.7 \%)$ & \\
\hline Dangour 2010 (OPAL) & $\mathrm{EPA}+\mathrm{DHA} 400 / 1000 \mathrm{mg}$ & 434 & $9(2.1 \%)$ & & Placebo & 433 & $8(1.8 \%)$ & \\
\hline Macchia 2013 (FORWARD) & $\begin{array}{c}\mathrm{EPA}+\mathrm{DHA} 850 \text { to } 882 \\
\mathrm{mg}\end{array}$ & 289 & $4(1.4 \%)$ & & Olive oil & 297 & $5(1.7 \%)$ & \\
\hline Manson 2019 (VITAL) & EPA + DHA 460/380 mg & 12933 & $371(2.9 \%)$ & $142(1.1 \%)$ & Placebo & 12938 & $381(2.9 \%)$ & $148(1.1 \%)$ \\
\hline Nilsen 2001 & $\begin{array}{c}\text { EPA + DHA } 850 \text { to } 882 \\
\text { mg (ratio 1:2) }\end{array}$ & 150 & $11(7.3 \%)$ & $8(5.3 \%)$ & Corn oil & 150 & $11(7.3 \%)$ & $8(5.3 \%)$ \\
\hline Raitt 2005 & EPA + DHA 756/540 mg & 100 & $4(4 \%)$ & $2(2 \%)$ & Placebo & 100 & $10(10 \%)$ & $5(5 \%)$ \\
\hline Rauch 2009 (OMEGA) & EPA + DHA 460/380 mg & 1919 & $88(4.6 \%)$ & $67(3.5 \%)$ & Olive oil & 1885 & $70(3.7 \%)$ & $51(2.7 \%)$ \\
\hline Valagussa 1999 (GISSI-P) & $\begin{array}{c}\text { EPA + DHA } 850 \text { to } 882 \\
\text { mg ( } \pm \text { vitamin E } 300 \mathrm{mg})\end{array}$ & 5666 & $472(8.3 \%)$ & $291(5.1 \%)$ & $\begin{array}{l}\text { Control }( \pm \\
\text { vitamin E } 300 \\
\mathrm{mg})\end{array}$ & 5668 & $545(9.6 \%)$ & $348(6.1 \%)$ \\
\hline Yokoyama 2007 (JELIS) & EPA $1800 \mathrm{mg}+$ statin & 9326 & $286(3.1 \%)$ & & Statin only & 9319 & $265(2.8 \%)$ & \\
\hline
\end{tabular}

CVD, cardiovascular disease; EPA, eicosapentaenoic acid; DHA, docosahexaenoic acid; ALA, alpha-linolenic acid. 
Table A4. Dose-response effects of statins and omega-3 supplementation on all-cause mortality and cardiovascular death.

\begin{tabular}{|c|c|c|c|c|c|}
\hline \multicolumn{3}{|c|}{ All-Cause Mortality } & \multicolumn{3}{|c|}{ Cardiovascular Death } \\
\hline Intervention & $\operatorname{RR}(95 \% \mathrm{CI})$ & $p$-Value & Intervention & RR (95\% CI) & $p$-Value \\
\hline Atorvastatin & $0.97(0.93-1.01)$ & 0.15 & Atorvastatin & $0.90(0.83-0.98)$ & 0.02 \\
\hline Pravastatin & $0.97(0.95-1.00)$ & 0.05 & Pravastatin & $0.96(0.94-0.98)$ & 0.001 \\
\hline Simvastatin & $0.93(0.80-1.07)$ & 0.32 & Simvastatin & $0.82(0.74-0.91)$ & $<0.001$ \\
\hline Rosuvastatin & $0.91(0.80-0.99)$ & 0.02 & Rosuvastatin & $0.97(0.91-1.03)$ & 0.36 \\
\hline Lovastatin & $0.93(0.40-1.30)$ & 0.28 & Fluvastatin & $0.92(0.86-0.98)$ & 0.01 \\
\hline $\begin{array}{c}\text { Omega-3 } \\
\text { supplementation }\end{array}$ & $0.98(0.95-1.01)$ & 0.11 & $\begin{array}{c}\text { Omega-3 } \\
\text { supplementation }\end{array}$ & $0.94(0.89-0.99)$ & 0.01 \\
\hline
\end{tabular}

$\mathrm{RR}$, relative risk; $\mathrm{CI}$, confidence interval. Data are presented as the effect of per increment in $10 \mathrm{mg}$ of statins and $1000 \mathrm{mg}$ of omega-3 supplementation. Bold font indicates statistical significance. 


\begin{tabular}{|c|c|c|c|c|c|}
\hline Study & Daily dose & $\begin{array}{l}\text { Follow-up } \\
\text { (years) }\end{array}$ & $\begin{array}{c}\text { Intervention c } \\
\mathrm{n} / \mathrm{N}\end{array}$ & $\underset{n / N}{\text { Comparison }}$ & RR $(95 \%$ Cl) \\
\hline \multicolumn{6}{|l|}{ Primary prevention } \\
\hline Blankenhorn 1993 (MARS) & Lovastatin $80 \mathrm{mg}$ & 2.2 & $1 / 123$ & 2/124 & $0.50(0.05-5.49)$ \\
\hline Colhoun 2004 (CARDS) & Atorvastatin $10 \mathrm{mg}$ & 3.9 & $61 / 1428$ & $82 / 1410$ & $0.73(0.53-1.01)$ \\
\hline Knopp 2006 (ASPEN) & Atorvastatin $10 \mathrm{mg}$ & 4 & 70/1211 & $68 / 1199$ & $1.02(0.74-1.41)$ \\
\hline Sever 2004 (ASCOT-LLA) & Atorvastatin $10 \mathrm{mg}$ & 5 & $185 / 5168$ & 212/5137 & $0.87(0.71-1.05)$ \\
\hline Teo2000(SCAT) & Simvastatin $10 \mathrm{mg}$ & 4 & $13 / 230$ & $6 / 230$ & $2.17(0.84-5.60)$ \\
\hline Wanner 2005 & Atorvastatin $20 \mathrm{mg}$ & 4 & $297 / 619$ & $320 / 636$ & $0.95(0.85-1.07)$ \\
\hline \multicolumn{5}{|c|}{ Subtotal (I-squared $=21.8 \%, p=0.270$ ) } & $0.92(0.81-1.04)$ \\
\hline \multicolumn{6}{|l|}{ Secondary prevention } \\
\hline Kjekshus 2007 (CORONA) & Rosuvastatin $10 \mathrm{mg}$ & 2.7 & $728 / 2514$ & $759 / 2497$ & $0.95(0.87-1.04)$ \\
\hline Koren 2004 (ALLIANCE) & Atorvastatin up to $80 \mathrm{mg}$ & 4.5 & $121 / 1217$ & $127 / 1225$ & $0.96(0.76-1.21)$ \\
\hline Liem 2002 (FLORIDA) & Fluvastatin $80 \mathrm{mg}$ & 1 & $7 / 265$ & $11 / 275$ & $0.66(0.26-1.68)$ \\
\hline \multicolumn{3}{|c|}{ Subtotal (I-squared $=0.0 \%, p=0.743$ ) } & & & $0.95(0.88-1.03)$ \\
\hline \multicolumn{6}{|l|}{ Mixed population } \\
\hline Amarenco 2006 (SPARCL) & Atorvastatin $80 \mathrm{mg}$ & 4.9 & $216 / 2365$ & $211 / 2366$ & $1.02(0.85-1.23)$ \\
\hline Arthros 2002 (GREACE) & Atorvastatin $10-80 \mathrm{mg}$ & 3.8 & $23 / 800$ & $40 / 800$ & $0.57(0.35-0.95)$ \\
\hline Davis 2002 (ALLHAT-LTT) & Pravastatin $40 \mathrm{mg}$ & 4.8 & $631 / 5170$ & $641 / 5185$ & $0.99(0.89-1.09)$ \\
\hline Emberson 2011 (MRC/BHF) & Simvastatin $40 \mathrm{mg}$ & 5 & $1328 / 10269$ & $1507 / 10267$ & $0.88(0.82-0.94)$ \\
\hline Fellstrom 2009 (AURORA) & Rosuvastatin $10 \mathrm{mg}$ & 3.8 & $636 / 1389$ & $660 / 1384$ & $0.96(0.89-1.04)$ \\
\hline Ford 2016 (WOSCOPS) & Pravastatin $40 \mathrm{mg}$ & 4.9 & 619/3302 & $674 / 3293$ & $0.92(0.83-1.01)$ \\
\hline Furberg 1994 (ACAPS) & Lovastatin $20-40 \mathrm{mg}$ (twarfarin $1 \mathrm{mg}$ ) & ) 2.8 & $1 / 460$ & $8 / 459<$ & $0.12(0.02-0.99)$ \\
\hline Furberg 1995 (PLAC-I \& II) & Pravastatin 20 or $40 \mathrm{mg}$ & 3 & $7 / 281$ & $11 / 278$ & $0.63(0.25-1.60)$ \\
\hline Makuuchi 2005 (PCABG) & Pravastatin $10-20 \mathrm{mg}$ & 4.5 & $6 / 152$ & $11 / 151$ & $0.54(0.21-1.43)$ \\
\hline Nakagawa 2004 (PCS) & Pravastatin $10 \mathrm{mg}$ & 5.4 & $17 / 54$ & $23 / 66$ & $0.90(0.54-1.51)$ \\
\hline Nakamura 2006 (MEGA) & Pravastatin $10-20 \mathrm{mg}$ & 5.3 & $55 / 3866$ & $79 / 3966$ & $0.71(0.51-1.00)$ \\
\hline Pedersen $1994(4 S)$ & Simvastatin $20 \mathrm{mg}$ & 5.4 & $182 / 2221$ & $256 / 2223$ & $0.71(0.59-0.85)$ \\
\hline Ridker 2008 (JUPITER) & Rosuvastatin $20 \mathrm{mg}$ & 1.9 & $198 / 8901$ & $247 / 8901$ & $0.80(0.67-0.96)$ \\
\hline Sawayama 2002 (FAST) & Pravastatin $10 \mathrm{mg}$ & 2 & $5 / 83$ & 9/81 & $0.54(0.19-1.55)$ \\
\hline Serruys 2002 (LIPS) & Fluvastatin $80 \mathrm{mg}$ & 3.9 & $36 / 844$ & $49 / 833$ & $0.73(0.48-1.10)$ \\
\hline Shepherd 2002 (PROSPER) & Pravastatin $40 \mathrm{mg}$ & 3.2 & $298 / 2891$ & $306 / 2913$ & $0.98(0.84-1.14)$ \\
\hline Takano 2013 (PEARL) & Pitavastatin $2 \mathrm{mg}$ & 3 & $27 / 288$ & $37 / 286$ & $0.72(0.45-1.16)$ \\
\hline Tavazzi 2008(GISSI-HF) & Rosuvastatin $10 \mathrm{mg}$ & 3.9 & $657 / 2285$ & $644 / 2289$ & $1.02(0.93-1.12)$ \\
\hline Tonkin 1998 (LIPID) & Pravastatin $40 \mathrm{mg}$ & 6.1 & $498 / 4512$ & 633/4502 & $0.78(0.70-0.88)$ \\
\hline Yokoi 2005 (ATHEROMA) & Pravastatin $10-20 \mathrm{mg}+$ diet & 3 & 1/142 & 2/146 & $0.51(0.05-5.61)$ \\
\hline & Rosuvastatin $10 \mathrm{mg}$ & 5.6 & $334 / 6361$ & $357 / 6344$ & $0.93(0.81-1.08)$ \\
\hline \multicolumn{5}{|c|}{ Subtotal (l-squared $=54.1 \%, p=0.002)$} & $0.89(0.84-0.94)$ \\
\hline \multicolumn{5}{|c|}{ Overall (I-squared $=43.8 \%, p=0.006$ ) } & $0.90(0.86-0.94)$ \\
\hline
\end{tabular}

Figure A1. Subgroup meta-analysis for the effect of statin use on primary or secondary prevention of all-cause mortality. Full black quadrilateral represents the point estimate of individual studies and blank with quadrilateral represents pooled estimates. RR, relative risk; CI, confidence interval. Pooled estimates for the overall study population and subgroups are highlighted with bold font. 


\begin{tabular}{|c|c|c|c|c|c|c|}
\hline Study & Daily dose & $\begin{array}{l}\text { Follow-up } \\
\text { (years) }\end{array}$ & $\begin{array}{l}\text { Intervention } \\
\mathrm{n} / \mathrm{N}(\%)\end{array}$ & $\begin{array}{c}\text { Comparison } \\
\mathrm{n} / \mathrm{N}(\%)\end{array}$ & & $\mathrm{RR}(95 \% \mathrm{Cl})$ \\
\hline $\begin{array}{l}\text { Atorvastatin } \\
\text { Amarenco } 2006 \\
\text { Colhoun 2004 } \\
\text { Knopp 2006 } \\
\text { Sever 2004 } \\
\text { Arthros 2002 } \\
\text { Koren 2004 } \\
\text { Wanner 2005 } \\
\text { Subtotal (I-squared = 22 }\end{array}$ & $\begin{array}{l}\text { Atorvastatin } 80 \mathrm{mg} \\
\text { Atorvastatin } 10 \mathrm{mg} \\
\text { Atorvastatin } 10 \mathrm{mg} \\
\text { Atorvastatin } 10 \mathrm{mg} \\
\text { Atorvastatin } 10-80 \mathrm{mg} \\
\text { Atorvastatin up to } 80 \mathrm{mg} \\
\text { Atorvastatin } 20 \mathrm{mg} \\
.6 \%, \boldsymbol{p}=\mathbf{0 . 2 5 7 )}\end{array}$ & $\begin{array}{l}4.9 \\
3.9 \\
4 \\
5 \\
3 \\
4.5 \\
4\end{array}$ & $\begin{array}{l}216 / 2365(9.1 \%) \\
61 / 1428(4.3 \%) \\
70 / 211(5.8 \%) \\
185 / 5168(3.6 \%) \\
23 / 500(2.9 \%) \\
121 / 1217(9) .9 \%) \\
297 / 619(48 \%)\end{array}$ & $\begin{array}{l}211 / 2366(8.9 \%) \\
82 / 1410(5.8 \%) \\
68 / 1199(5.7 \%) \\
21215137(4) .1 \%) \\
40 / 800(5 \%) \\
127 / 1225(10.4 \%) \\
320 / 636(50.3 \%)\end{array}$ & - & $\begin{array}{l}1.02(0.85-1.23) \\
0.73(0.53-1.01) \\
1.02(0.74-1.41) \\
0.87(0.71-1.05) \\
0.57(0.05-0.95) \\
0.96(0.76-1.21) \\
0.95(0.85-1.07) \\
0.92(0.84-1.01)\end{array}$ \\
\hline $\begin{array}{l}\text { Pravastatin } \\
\text { Davis 2002 } \\
\text { Ford 2016 } \\
\text { Nakagawa 2004 } \\
\text { Nakamura 2006 } \\
\text { Tonin 1998 } \\
\text { Furberg 1995 } \\
\text { Makuchich 2005 } \\
\text { Sawayama 2002 } \\
\text { Sheyherd 2002 } \\
\text { Yokion 2005 } \\
\text { Subtotal (I-squared = } 39\end{array}$ & $\begin{array}{l}\text { Pravastatin } 40 \mathrm{mg} \\
\text { Pravastatin } 40 \mathrm{mg} \\
\text { Pravastatin } 10 \mathrm{mg} \\
\text { Pravastatin } 10-20 \mathrm{mg} \\
\text { Pravastatit } 40 \mathrm{mg} \\
\text { Pravastatin } 20 \text { or } 40 \mathrm{mg} \\
\text { Pravastatin } 10-20 \mathrm{mg} \\
\text { Pravastatin } 10 \mathrm{mg} \\
\text { Pravastatitin } 40 \mathrm{mg} \\
\text { Pravastatin } 10-20 \mathrm{mg} \text { + diet } \\
\mathbf{7 \%} \%, \boldsymbol{p}=\mathbf{0 . 0 9 3 )}\end{array}$ & $\begin{array}{l}4.8 \\
4.9 \\
5.4 \\
5.3 \\
6.1 \\
3 \\
4.5 \\
2.5 \\
3.2 \\
3\end{array}$ & $\begin{array}{l}631 / 5170(12.2 \%) \\
61933302(18.7 \%) \\
17 / 54(31.5 \%) \\
55 / 3866(1.4 \%) \\
498 / 4512(11 \%) \\
71281(2.5 \%) \\
6 / 152(3.9 \%) \\
5 / 83(6 \%) \\
298 / 2891(10.3 \%) \\
1 / 142(0.7 \%)\end{array}$ & $\begin{array}{l}641 / 5185(12.4 \%) \\
6743293(20.5 \%) \\
23 / 66(34.8 \%) \\
796 / 3966(2 \%) \\
633 / 4502(14.1 \%) \\
11 / 278(4 \%) \\
11 / 151(7.3 \%) \\
9 / 81(11.1 \%) \\
306 / 2913(10.5 \%) \\
21146(1.4 \%)\end{array}$ & $\underset{+\infty}{\stackrel{0}{*}+\infty}$ & $\begin{array}{l}0.99(0.89-1.09) \\
0.92(0.83-1.01) \\
0.90(0.54-1.51) \\
0.71(0.51-1.00) \\
0.78(0.70-0.88) \\
0.63(0.25-1.60) \\
0.54(0.21-1.43) \\
0.54(0.19-1.55) \\
0.98(0.84-1.14) \\
0.51(0.05-5.61) \\
0.89(0.81-0.97)\end{array}$ \\
\hline $\begin{array}{l}\text { Simvastatin } \\
\text { Pedersen } 1994 \\
\text { Emberson } 2011 \\
\text { Teo 2000 } \\
\text { Subtotal (I-squared = 76 }\end{array}$ & $\begin{array}{l}\text { Simvastatin } 20 \mathrm{mg} \\
\text { Simvastatin } 40 \mathrm{mg} \\
\text { Simvastatin } 10 \mathrm{mg} \\
.0 \%, p=0.016)\end{array}$ & $\begin{array}{l}5.4 \\
5 \\
4\end{array}$ & $\begin{array}{l}182 / 2221(8.2 \%) \\
1328 / 10269(12.9 \%) \\
13 / 230(5.7 \%)\end{array}$ & $\begin{array}{l}256 / 2223(11.5 \%) \\
1507110267(14.7 \%) \\
6 / 230(2.6 \%)\end{array}$ & & $\begin{array}{l}0.71(0.59-0.85) \\
0.88(0.82-0.94) \\
2.17(0.84-5.60) \\
0.85(0.66-1.09)\end{array}$ \\
\hline $\begin{array}{l}\text { Rosuvastatin } \\
\text { Ridker 2008 } \\
\text { Tavazzi 2008 } \\
\text { Yusuf } 2016 \\
\text { Fellstrom } 2009 \\
\text { Kjekshus 2007 } \\
\text { Subtotal (l-squared = } 99\end{array}$ & $\begin{array}{l}\text { Rosuvastatin } 20 \mathrm{mg} \\
\text { Rosuvastatin } 10 \mathrm{mg} \\
\text { Rosuvastatin } 10 \mathrm{mg} \\
\text { Rosuvastatin } 10 \mathrm{mg} \\
\text { Rosuvastatin } 10 \mathrm{mg} \\
\mathbf{4} \%, \boldsymbol{p}=\mathbf{0 . 2 2 5 )}\end{array}$ & $\begin{array}{l}1.9 \\
3.9 \\
5.6 \\
3.8 \\
2.7\end{array}$ & $\begin{array}{l}198 / 8901(2.2 \%) \\
657 / 2285(28.8 \%) \\
334 / 6361(5.3 \%) \\
636 / 1389(45.8 \%) \\
728 / 2514(29 \%)\end{array}$ & $\begin{array}{l}247 / 8901(2.8 \%) \\
644 / 2289(28.1 \%) \\
357 / 6344(5.6 \%) \\
660 / 1384(47.7 \%) \\
759 / 2497(30.4 \%)\end{array}$ & 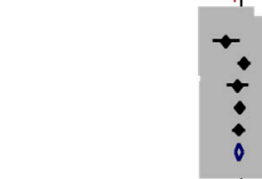 & $\begin{array}{l}0.80(0.67-0.96) \\
1.02(0.93-1.12) \\
0.93(0.81-1.08) \\
0.96(0.89-1.04) \\
0.95(0.87-1.04) \\
\mathbf{0 . 9 6}(0.90-1.01)\end{array}$ \\
\hline $\begin{array}{l}\text { Lovastatin } \\
\text { Furberg } 1994 \\
\text { Blankenhorn } 1993 \\
\text { Subtotal (I-squared }=0 .\end{array}$ & $\begin{array}{l}\text { Lovastatin } 20-40 \mathrm{mg} \text { ( } \pm \text { warfarin) } \\
\text { Lovastatin } 80 \mathrm{mg} \\
6, p=0.379)\end{array}$ & $\begin{array}{l}2.8 \\
2.2\end{array}$ & $\begin{array}{l}1 / 460(0.2 \%) \\
1 / 123(0.8 \%)\end{array}$ & $\begin{array}{l}8 / 459(1.7 \%) \\
2 / 124(1.6 \%)\end{array}$ & $\longrightarrow$ & $\begin{array}{l}0.12(0.02-0.99) \\
0.50(0.05-5.49) \\
0.23(0.05-1.09\end{array}$ \\
\hline $\begin{array}{l}\text { Fluvastatin } \\
\quad \text { Liem } 2002 \\
\text { Serrysy 2002 } \\
\text { Subtotal (I-squared }=0 .\end{array}$ & $\begin{array}{l}\text { Fluvastatin } 80 \mathrm{mg} \\
\text { Fluvastatin } 80 \mathrm{mg} \\
6, p=0.858)\end{array}$ & $\begin{array}{l}1 \\
3.9\end{array}$ & $\begin{array}{l}7 / 265(2.6 \%) \\
36 / 844(4.3 \%)\end{array}$ & $\begin{array}{l}11 / 275(4 \%) \\
49 / 833(5.9 \%)\end{array}$ & & $\begin{array}{l}0.66(0.26-1.68) \\
0.73(0.48-1.10) \\
0.71(0.49-1.05)\end{array}$ \\
\hline $\begin{array}{l}\text { Pitavastatin } \\
\text { Takano } 2013 \\
\text { Subtotal }\end{array}$ & Pitavastatin $2 \mathrm{mg}$ & 3 & $27 / 288(9.4 \%)$ & $37286(12.9 \%)$ & & $\begin{array}{l}0.72(0.45-1.16) \\
0.72(0.45-1.16)\end{array}$ \\
\hline Overall (I-squared $=43$. & $\%, p=0.006)$ & & & & of & $0.90(0.86-0.94)$ \\
\hline
\end{tabular}

Figure A2. Subgroup meta-analysis for the effect of different statins on all-cause mortality. Full black quadrilateral represents the point estimate of individual studies and blank with quadrilateral represents pooled estimates. RR, relative risk; CI, confidence interval. Pooled estimates for the overall study population and subgroups are highlighted with bold font. 


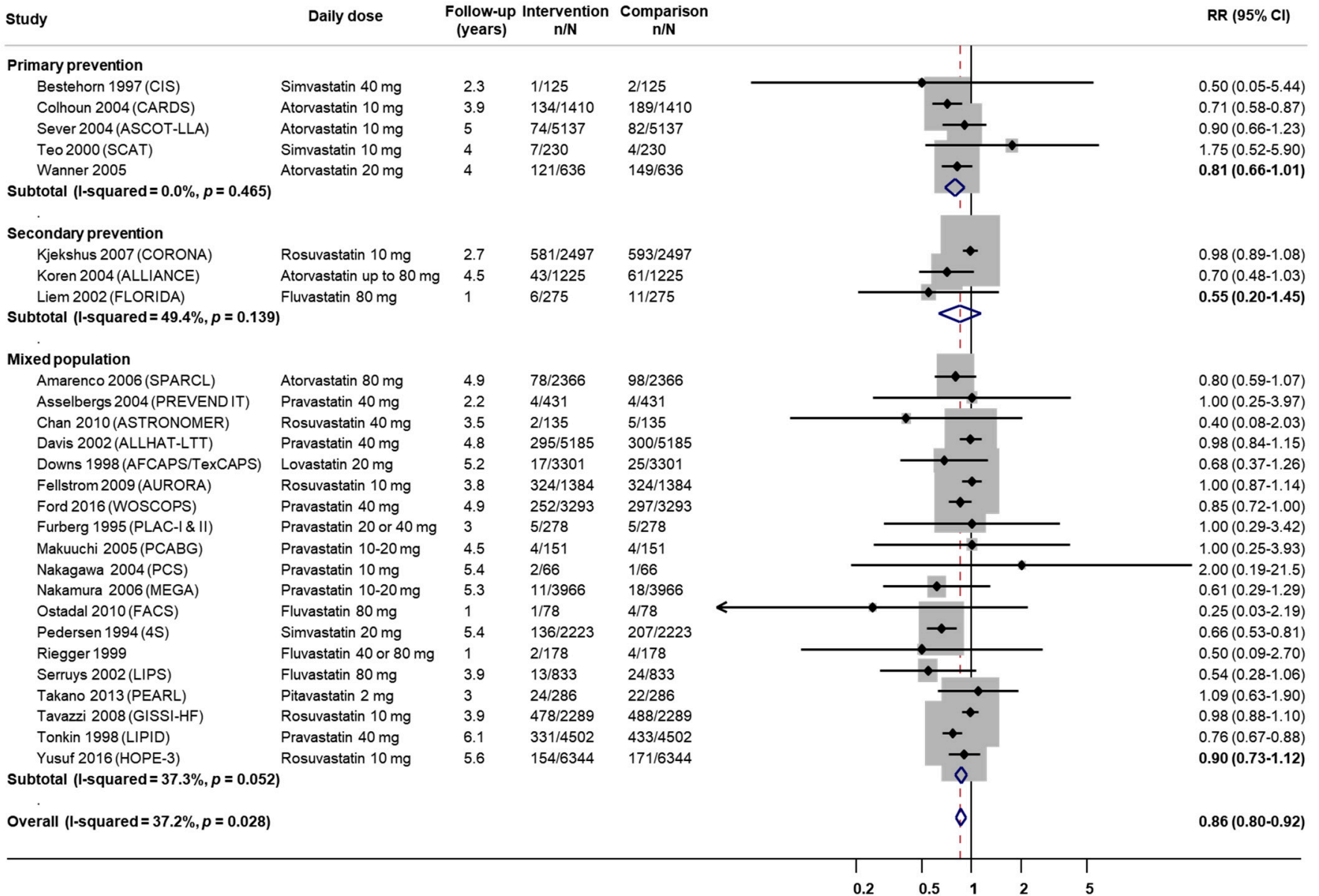

Figure A3. Subgroup meta-analysis for the effect of statin use on primary or secondary prevention of cardiovascular disease death. Full black quadrilateral represents the point estimate of individual studies and blank with quadrilateral represents pooled estimates. RR, relative risk; CI, confidence interval. Pooled estimates for the overall study population and subgroups are highlighted with bold font. 


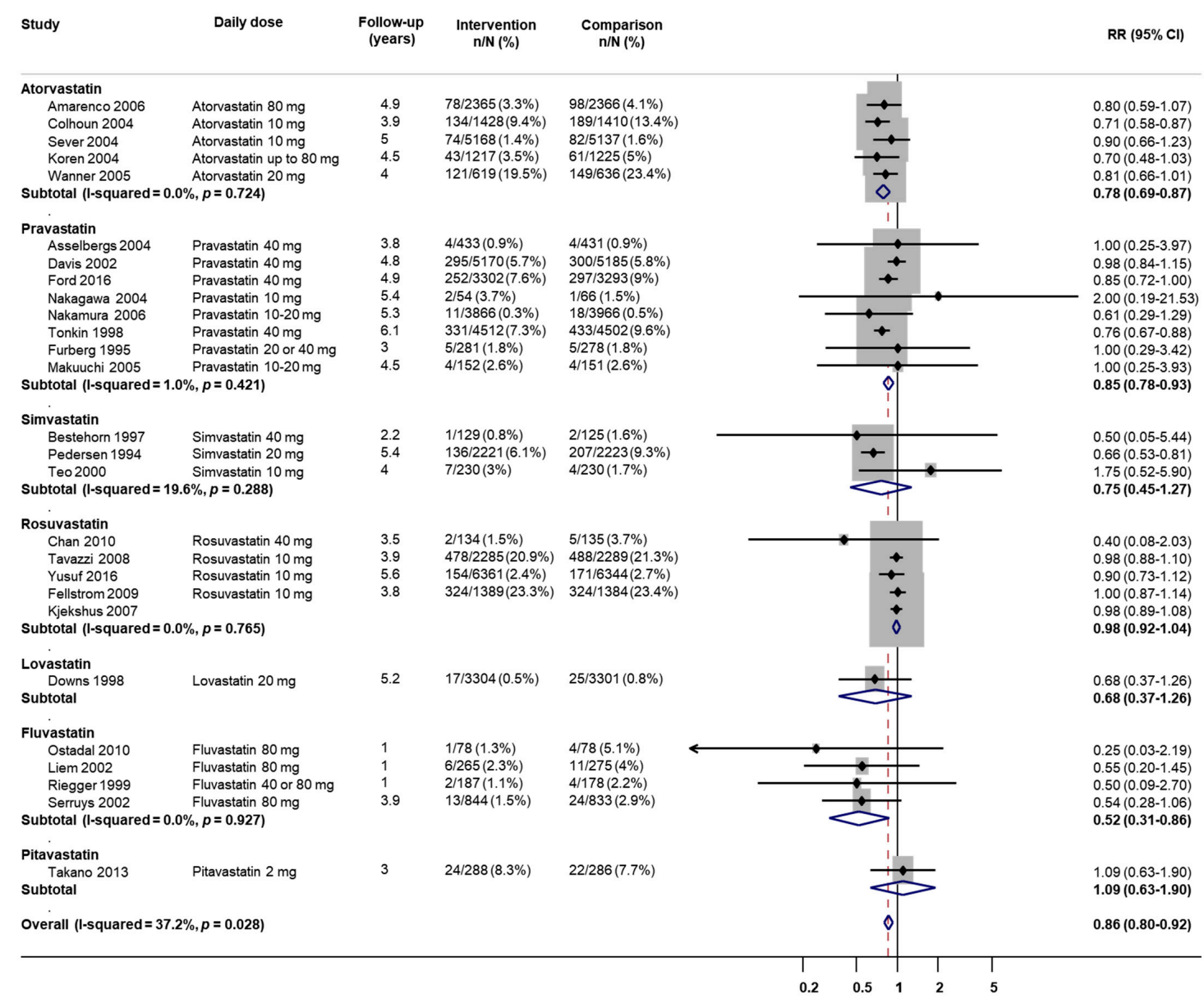

Figure A4. Subgroup meta-analysis for the effect of different statins on cardiovascular disease death. Full black quadrilateral represents the point estimate of individual studies and blank with quadrilateral represents pooled estimates. RR, relative risk; CI, confidence interval. Pooled estimates for the overall study population and subgroups are highlighted with bold font. 


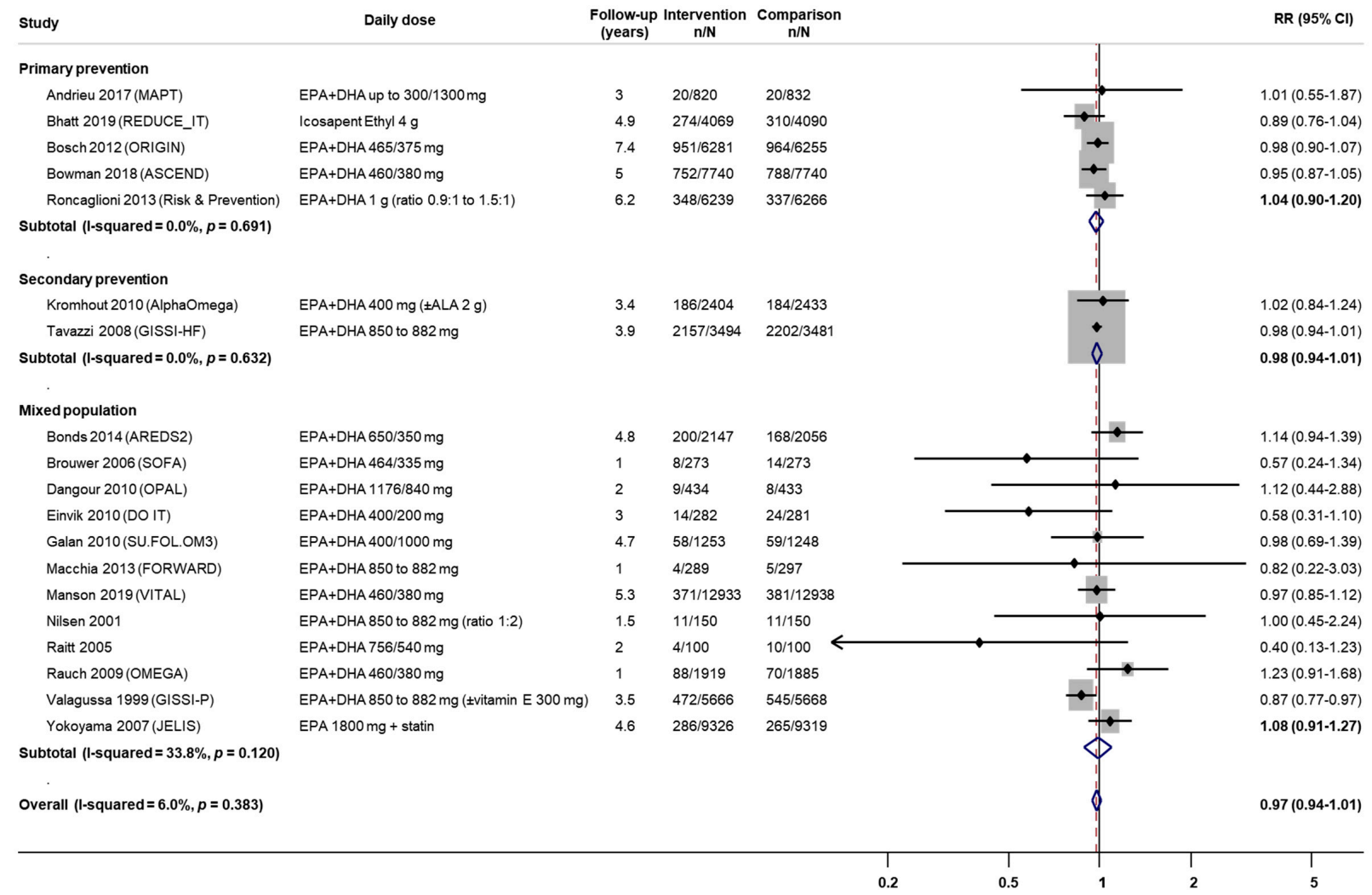

Figure A5. Subgroup meta-analysis for the effect of omega-3 supplementation on primary or secondary prevention of all-cause mortality. Full black quadrilateral represents the point estimate of individual studies and blank with quadrilateral represents pooled estimates. RR, relative risk; CI, confidence interval; EPA, eicosapentaenoic acid; DHA, docosahexaenoic acid. ALA, alpha-linolenic acid. Pooled estimates for the overall study population and subgroups are highlighted with bold font 


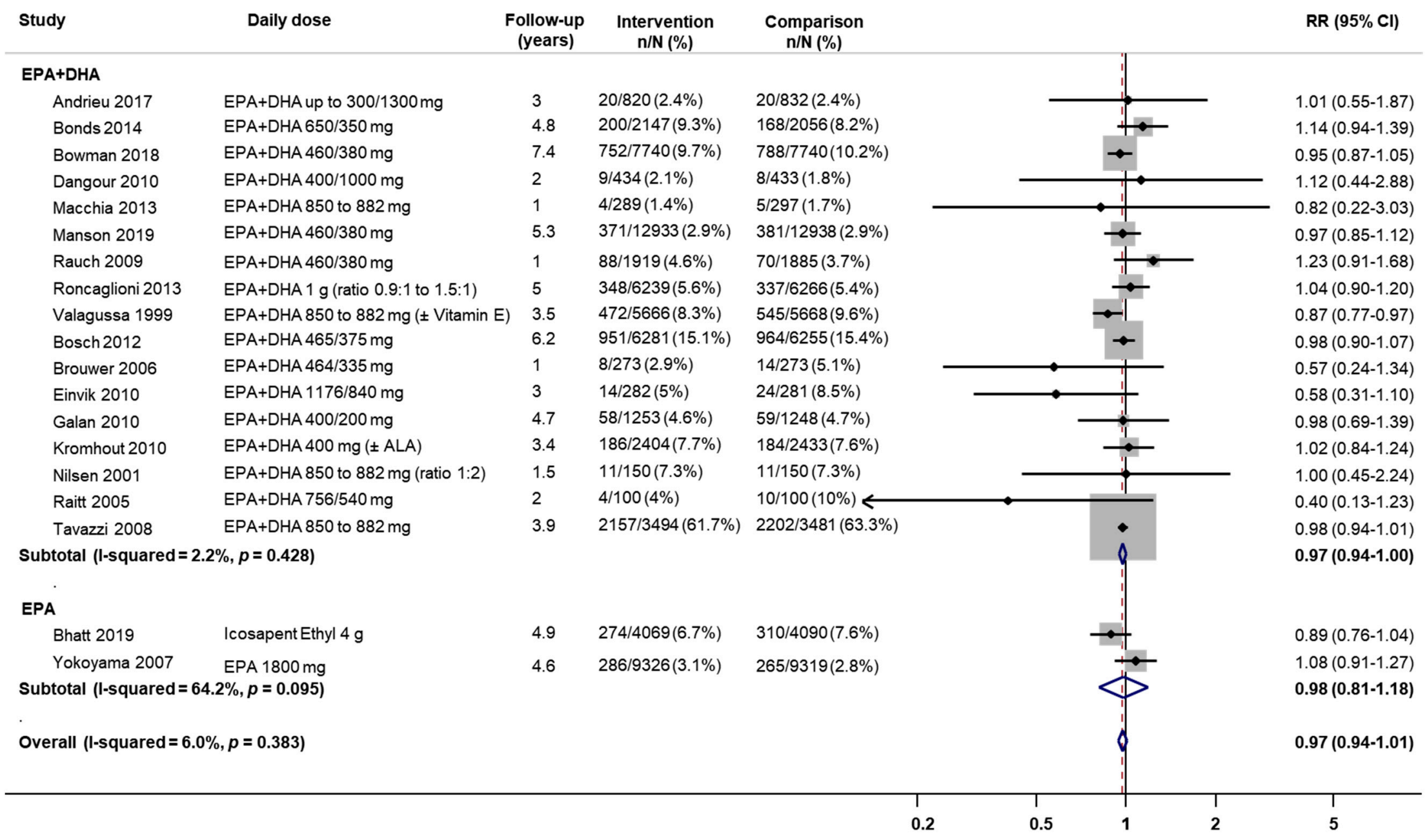

Figure A6. Subgroup meta-analysis for the effect of different omega-3 supplementation types on all-cause mortality. Full black quadrilateral represents the point estimate of individual studies and blank with quadrilateral represents pooled estimates. RR, relative risk; CI, confidence interval; EPA, eicosapentaenoic acid; DHA, docosahexaenoic acid. ALA, alpha-linolenic acid. Pooled estimates for the overall study population and subgroups are highlighted with bold font. 


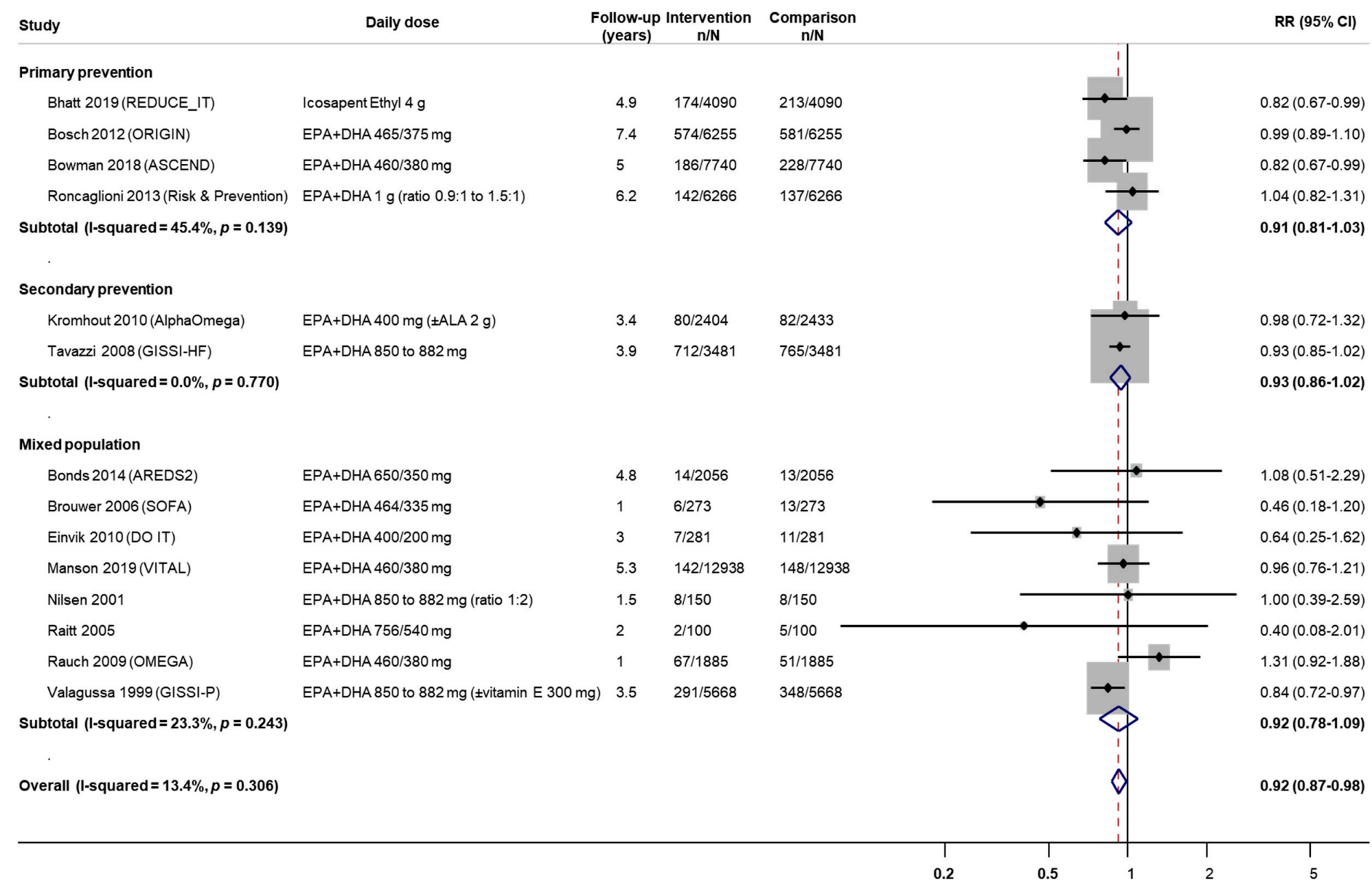

Figure A7. Subgroup meta-analysis for the effect of omega-3 supplementation on primary or secondary prevention of cardiovascular disease death. Full black quadrilateral represents the point estimate of individual studies and blank with quadrilateral represents pooled estimates. RR, relative risk; CI, confidence interval; EPA, eicosapentaenoic acid; DHA, docosahexaenoic acid. ALA, alpha-linolenic acid. Pooled estimates for the overall study population and subgroups are highlighted with bold font. 


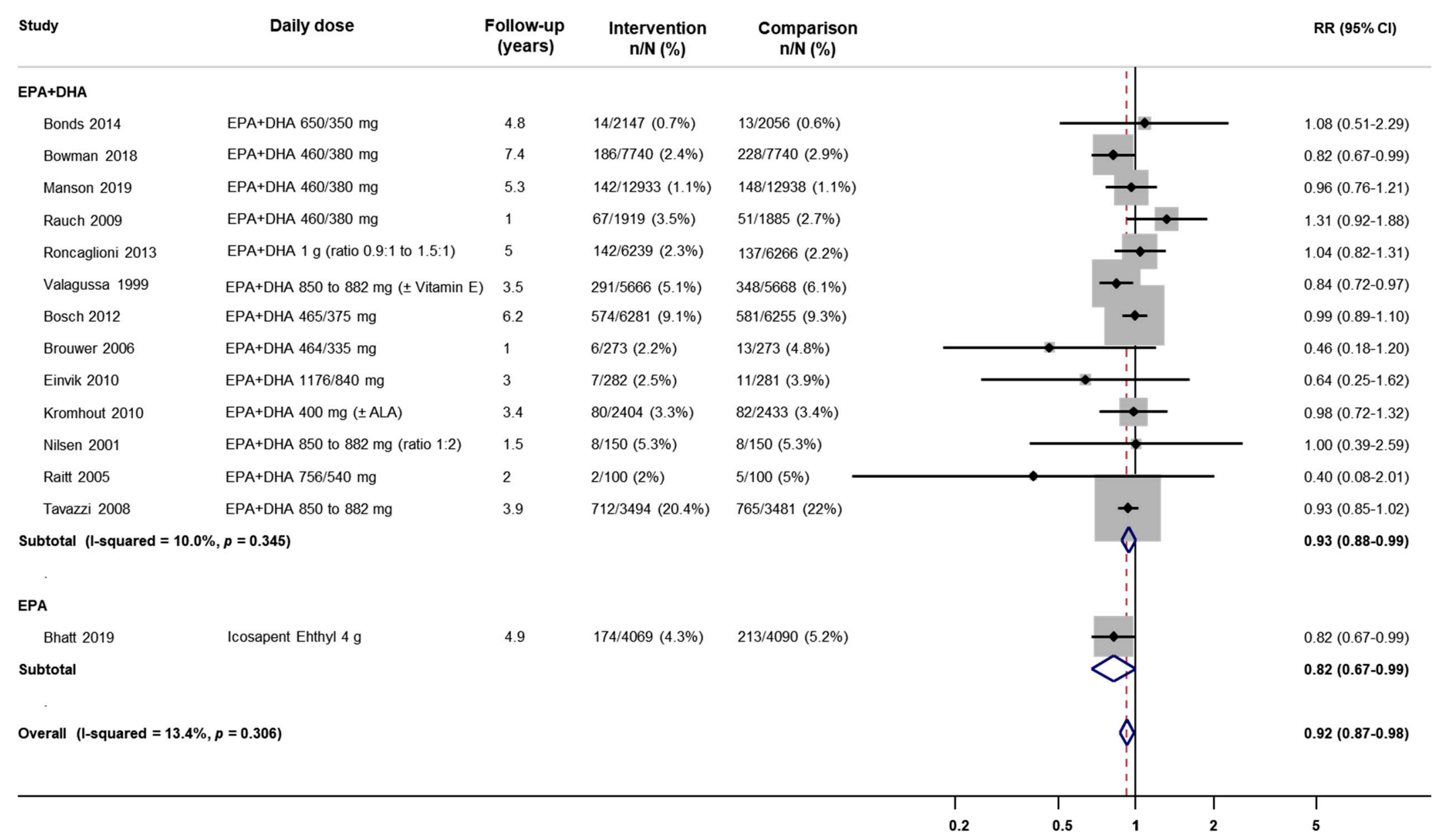

Figure A8. Subgroup meta-analysis for the effect of different omega-3 supplementation types on cardiovascular disease death. Full black quadrilateral represents the point estimate of individual studies and blank with quadrilateral represents pooled estimates. RR, relative risk; CI, confidence interval; EPA, eicosapentaenoic acid; DHA, docosahexaenoic acid. ALA, alpha-linolenic acid. Pooled estimates for the overall study population and subgroups are highlighted with bold font. 
A
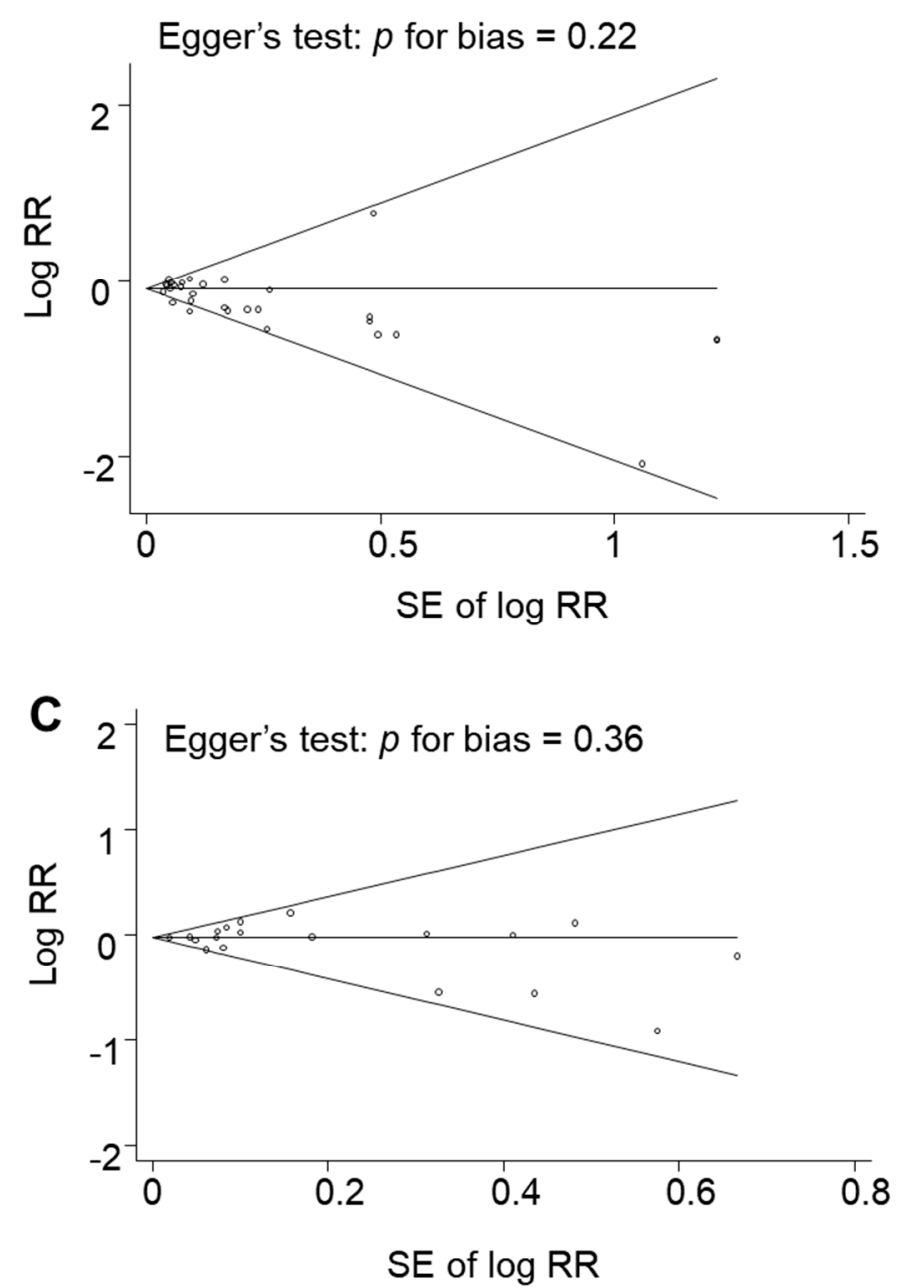

B
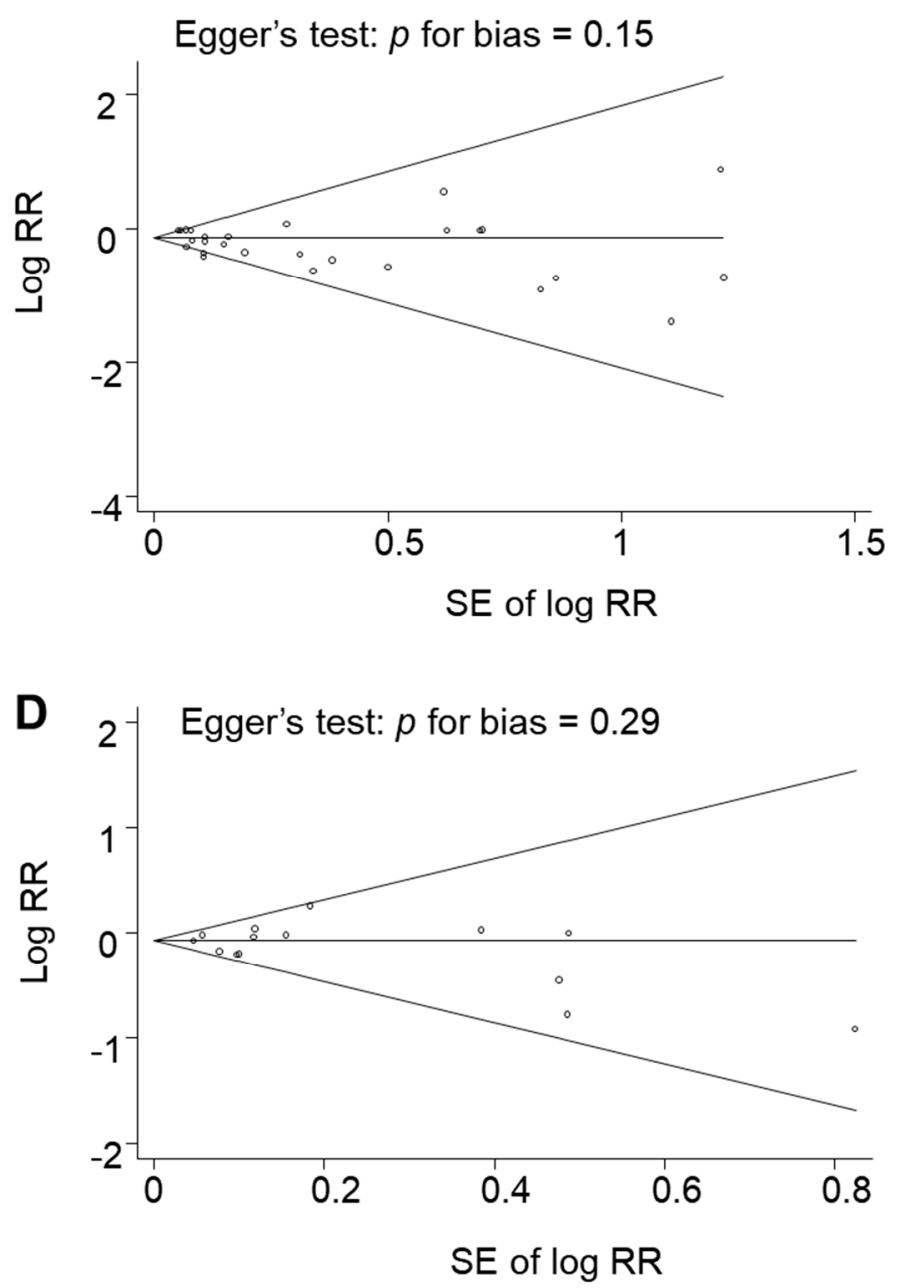

Figure A9. Funnel plot analysis to detect publication bias: (A) statins and all-cause mortality; (B) statins and cardiovascular disease death; (C) omega-3 supplementation and all-cause mortality; and (D) omega-3 supplementation and cardiovascular disease death). RR, relative risk; SE, standard error. 

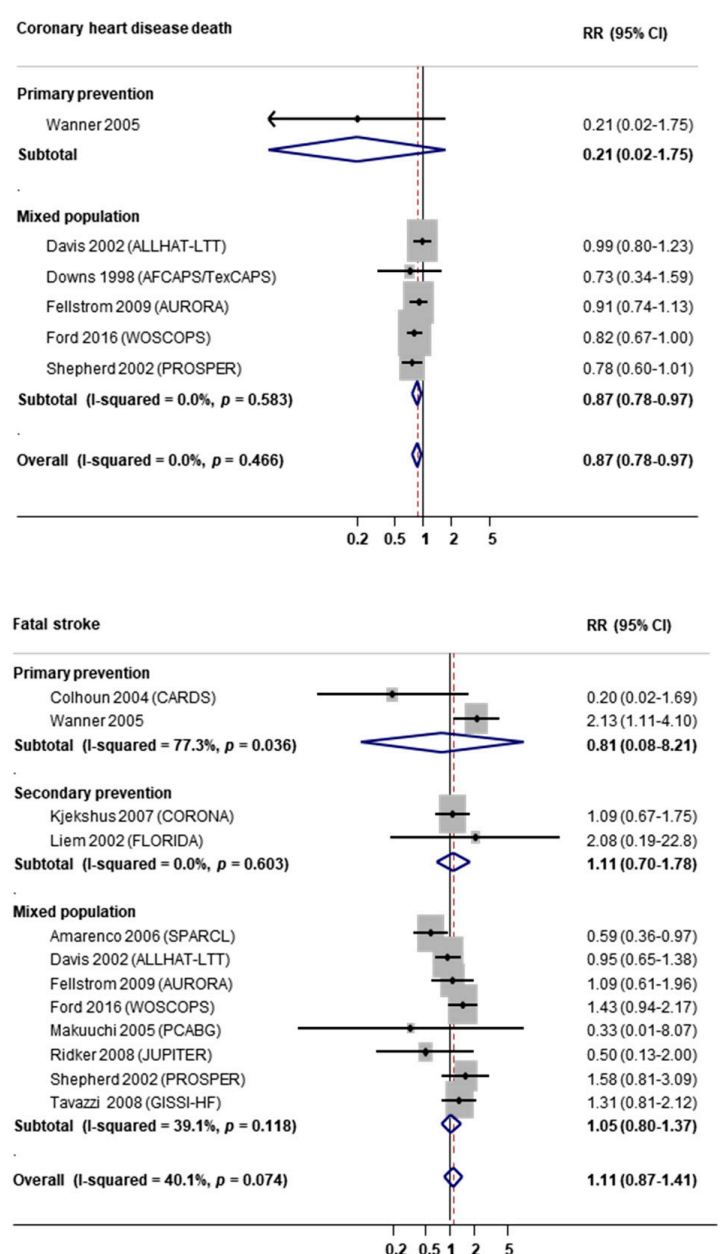

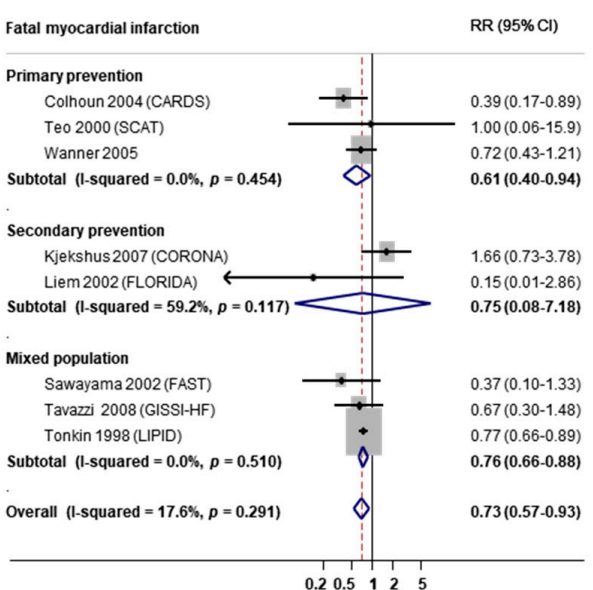

0.20 .5125

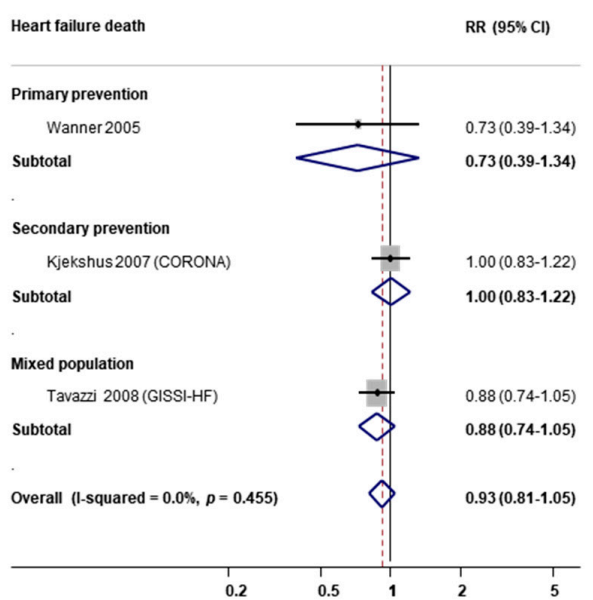

Figure A10. Subgroup meta-analysis for the effect of statins on specific causes of cardiovascular disease death. Full black quadrilateral represents the point estimate of individual studies and blank with quadrilateral represents pooled estimates. RR, relative risk; CI, confidence interval. Pooled estimates for the overall study population and subgroups are highlighted with bold font. 


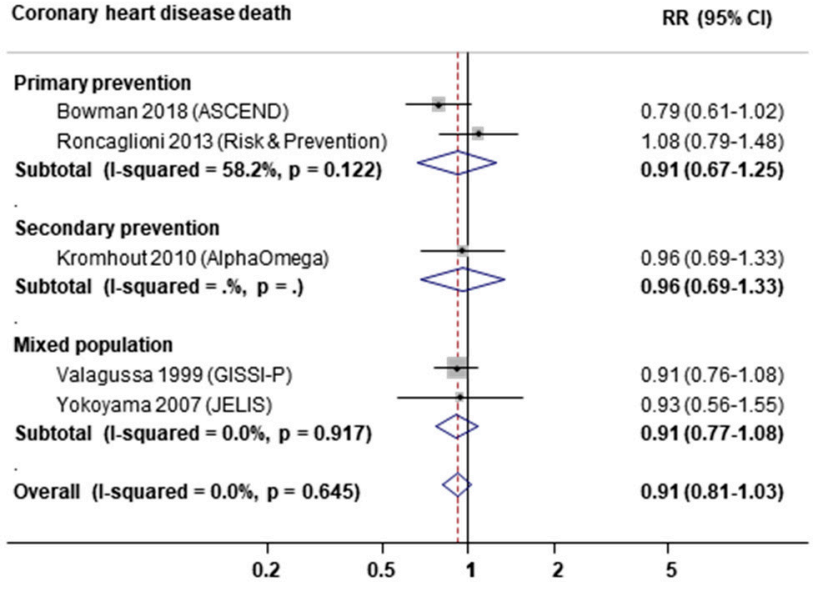

\begin{tabular}{|c|c|}
\hline Fatal stroke & RR $(95 \% \mathrm{Cl})$ \\
\hline \multicolumn{2}{|l|}{ Primary prevention } \\
\hline Bowman 2018 (ASCEND) & $0.95(0.60-1.50)$ \\
\hline Subtotal & $0.95(0.60-1.50)$ \\
\hline \multirow{2}{*}{\multicolumn{2}{|c|}{ Secondary prevention }} \\
\hline & \\
\hline Tavazzi 2008 (GISSI-HF) & $1.13(0.76-1.69)$ \\
\hline Subtotal & $1.13(0.76-1.69)$ \\
\hline . & \\
\hline Overall (I-squared $=0.0 \%, p=0.565$ ) & $1.05(0.77-1.42)$ \\
\hline 0.2 & 5 \\
\hline
\end{tabular}
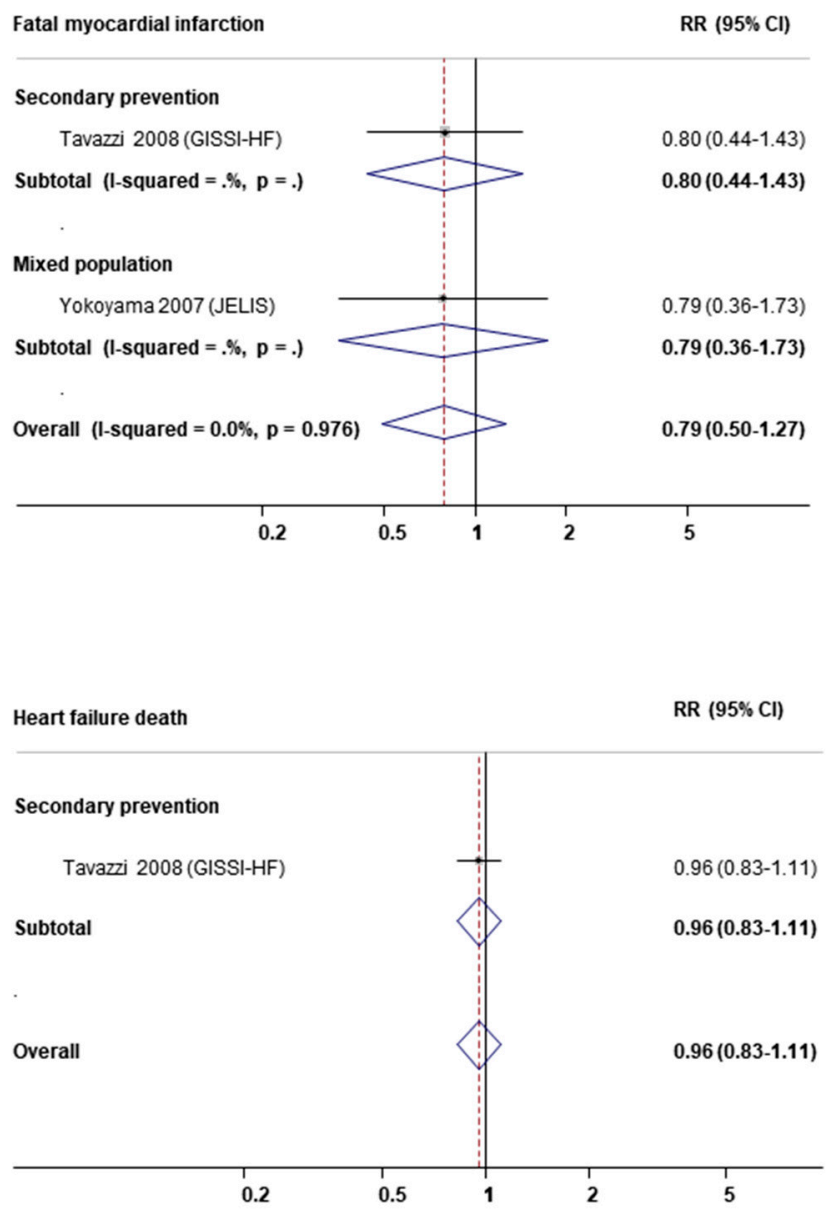

Figure A11. Subgroup meta-analysis for the effect of omega-3 supplementation on specific causes of cardiovascular disease death. Full black quadrilateral represents the point estimate of individual studies and blank with quadrilateral represents pooled estimates. RR, relative risk; CI, confidence interval. Pooled estimates for the overall study population and subgroups are highlighted with bold font. 
A

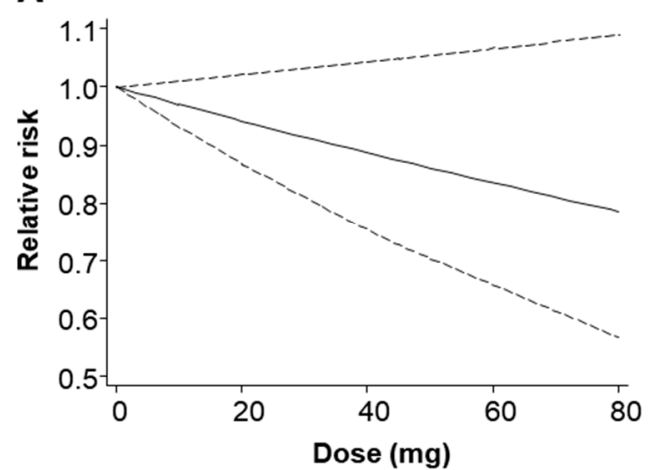

D

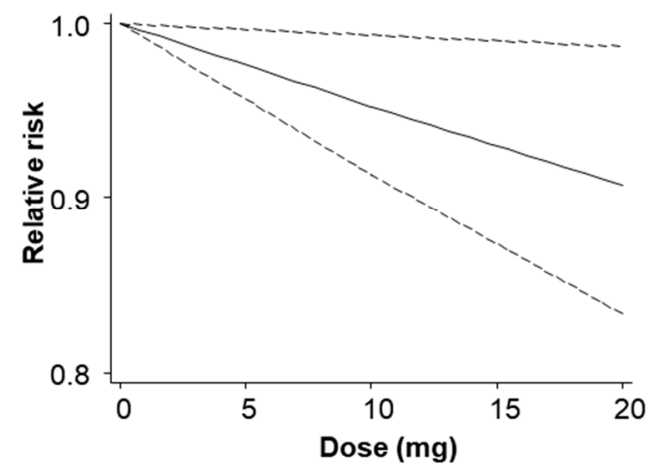

B

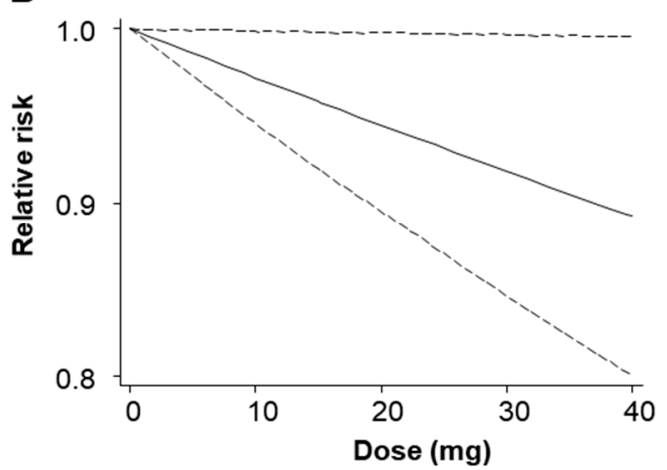

E

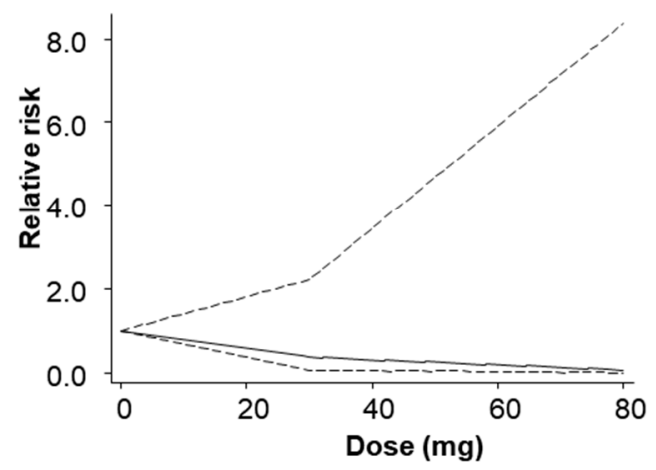

C

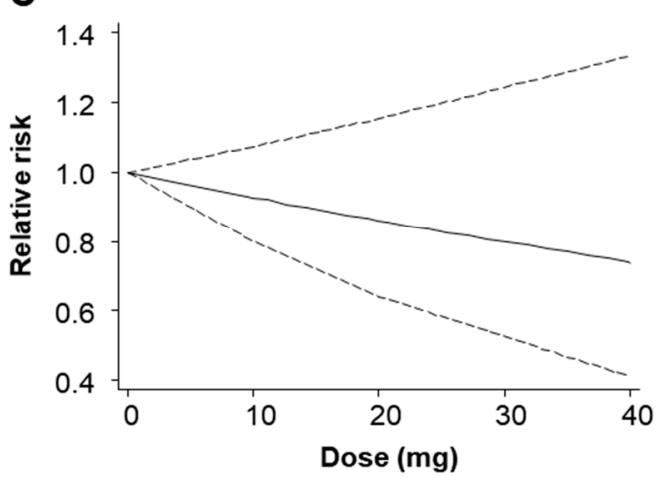

F

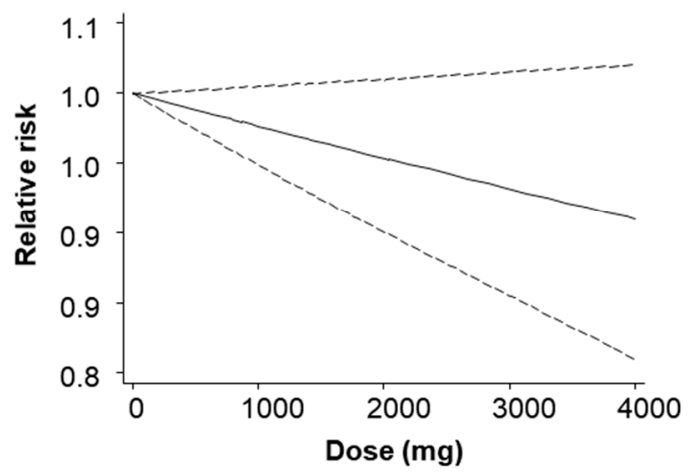

Figure A12. Dose-response meta-analysis of the association between (A) atorvastatin, (B) pravastatin, (C) simvastatin, (D) rosuvastain, (E) lovastatin, and (F) omega-3 supplementation with all-cause mortality. 
A

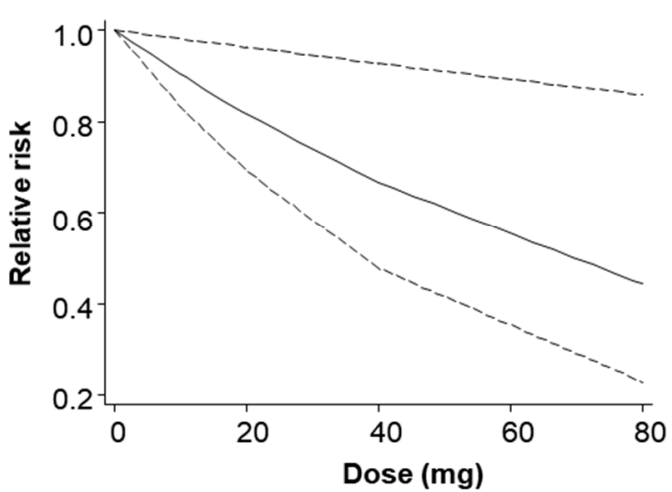

D

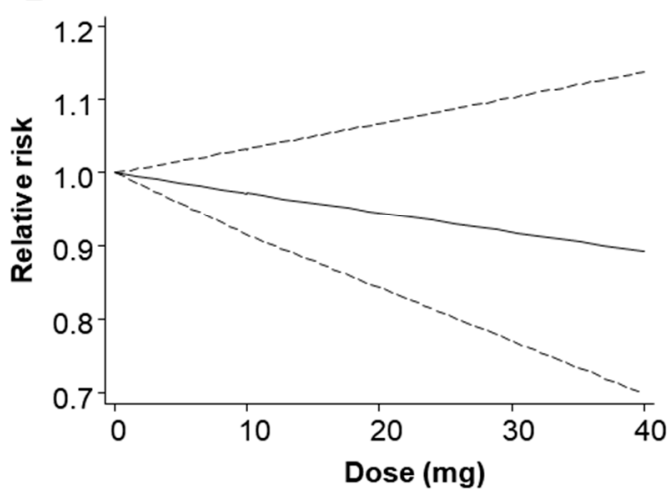

B

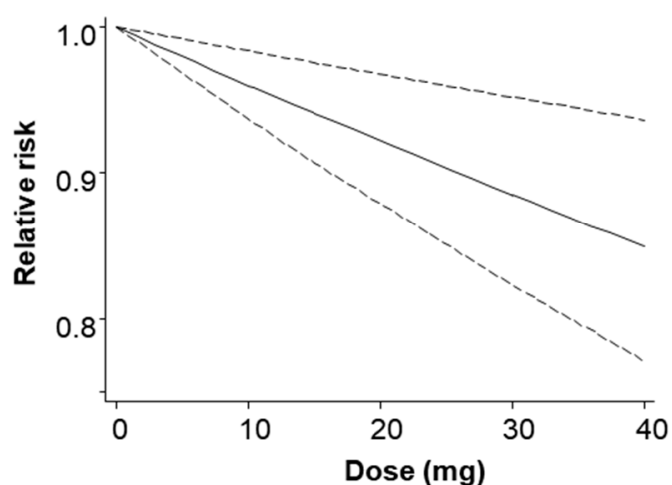

E

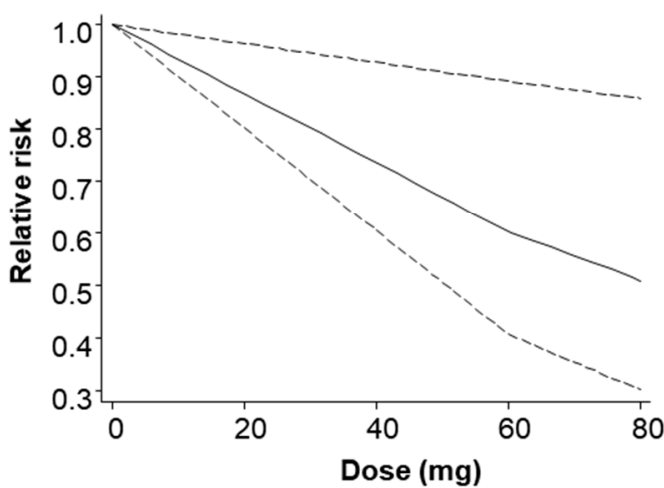

C

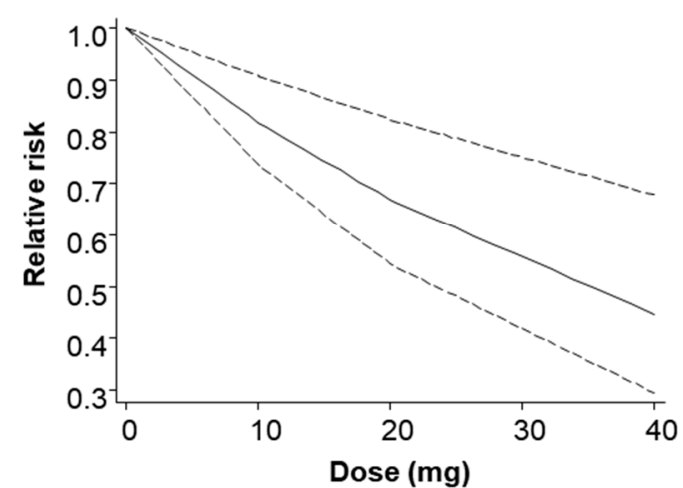

F

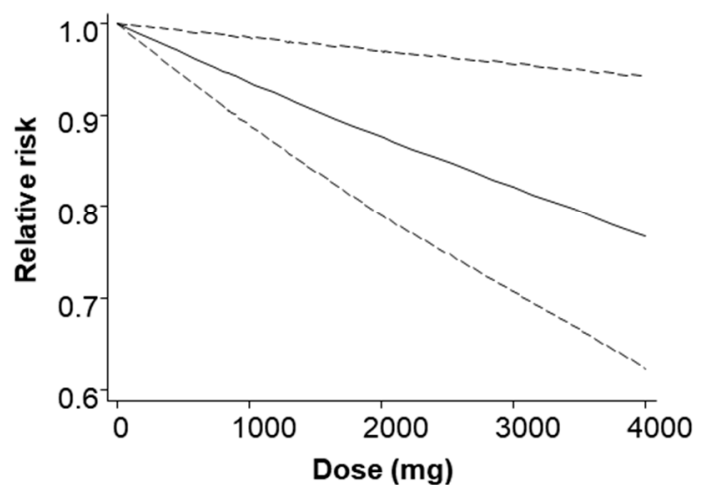

Figure A13. Dose-response meta-analysis of the association between (A) atorvastatin, (B) pravastatin, (C) simvastatin, (D) rosuvastain, (E) fluvastatin, and (F) omega-3 supplementation with cardiovascular death. 


\section{References}

1. GBD Causes of Death Collaborators. Global, regional, and national age-sex-specific mortality for 282 causes of death in 195 countries and territories, 1980-2017: A systematic analysis for the Global Burden of Disease Study 2017. Lancet 2018, 392, 1736-1788. [CrossRef]

2. Khavjou, O.; Phelps, D.; Leib, A.; RITI International. Projections of Cardiovascular Disease Prevalence and Costs: 2015-2035. Available online: https://healthmetrics.heart.org/wp-content/uploads/2017/10/Projectionsof-Cardiovascular-Disease.pdf (accessed on 16 February 2020).

3. GBD DALYs Hale Collaborators. Global, regional, and national disability-adjusted life-years (DALYs) for 359 diseases and injuries and healthy life expectancy (HALE) for 195 countries and territories, 1990-2017: A systematic analysis for the Global Burden of Disease Study 2017. Lancet 2018, 392, 1859-1922. [CrossRef]

4. Muller, C.J.; Noonan, C.J.; MacLehose, R.F.; Stoner, J.A.; Lee, E.T.; Best, L.G.; Calhoun, D.; Jolly, S.E.; Devereux, R.B.; Howard, B.V. Trends in cardiovascular disease morbidity and mortality in American Indians over 25 years: The Strong Heart Study. J. Am. Heart Assoc. 2019, 8, e012289. [CrossRef]

5. Fellstrom, B.C.; Jardine, A.G.; Schmieder, R.E.; Holdaas, H.; Bannister, K.; Beutler, J.; Chae, D.W.; Chevaile, A.; Cobbe, S.M.; Gronhagen-Riska, C.; et al. Rosuvastatin and cardiovascular events in patients undergoing hemodialysis. N. Engl. J. Med. 2009, 360, 1395-1407. [CrossRef]

6. Arnett, D.K.; Blumenthal, R.S.; Albert, M.A.; Buroker, A.B.; Goldberger, Z.D.; Hahn, E.J.; Himmelfarb, C.D.; Khera, A.; Lloyd-Jones, D.; McEvoy, J.W.; et al. 2019 ACC/AHA guideline on the primary prevention of cardiovascular disease: A report of the American College of Cardiology/American Heart Association Task Force on clinical practice guidelines. Circulation 2019, 140, e596-e646. [CrossRef]

7. Piepoli, M.F.; Hoes, A.W.; Agewall, S.; Albus, C.; Brotons, C.; Catapano, A.L.; Cooney, M.T.; Corra, U.; Cosyns, B.; Deaton, C.; et al. 2016 European Guidelines on cardiovascular disease prevention in clinical practice: The sixth joint task force of the European Society of Cardiology and Other Societies on Cardiovascular Disease Prevention in Clinical Practice (constituted by representatives of 10 societies and by invited experts) developed with the special contribution of the European Association for Cardiovascular Prevention \& Rehabilitation (EACPR). Eur. Heart J. 2016, 37, 2315-2381. [CrossRef]

8. Yebyo, H.G.; Aschmann, H.E.; Kaufmann, M.; Puhan, M.A. Comparative effectiveness and safety of statins as a class and of specific statins for primary prevention of cardiovascular disease: A systematic review, meta-analysis, and network meta-analysis of randomized trials with 94,283 participants. Am. Heart J. 2019, 210, 18-28. [CrossRef]

9. Eckel, R.H.; Jakicic, J.M.; Ard, J.D.; de Jesus, J.M.; Houston Miller, N.; Hubbard, V.S.; Lee, I.M.; Lichtenstein, A.H.; Loria, C.M.; Millen, B.E.; et al. 2013 AHA/ACC guideline on lifestyle management to reduce cardiovascular risk: A report of the American College of Cardiology/American Heart Association Task Force on Practice Guidelines. Circulation 2014, 129, S76-S99. [CrossRef]

10. Alexander, D.D.; Miller, P.E.; Van Elswyk, M.E.; Kuratko, C.N.; Bylsma, L.C. A meta-analysis of randomized controlled trials and prospective cohort studies of eicosapentaenoic and docosahexaenoic long-chain omega-3 fatty acids and coronary heart disease risk. Mayo Clin. Proc. 2017, 92, 15-29. [CrossRef]

11. Mohebi-Nejad, A.; Bikdeli, B. Omega-3 supplements and cardiovascular diseases. Tanaffos 2014, 13, 6-14.

12. Siscovick, D.S.; Barringer, T.A.; Fretts, A.M.; Wu, J.H.; Lichtenstein, A.H.; Costello, R.B.; Kris-Etherton, P.M.; Jacobson, T.A.; Engler, M.B.; Alger, H.M.; et al. Omega-3 polyunsaturated fatty acid (fish oil) supplementation and the prevention of clinical cardiovascular disease: A science advisory from the American Heart Association. Circulation 2017, 135, e867-e884. [CrossRef] [PubMed]

13. Abdelhamid, A.S.; Brown, T.J.; Brainard, J.S.; Biswas, P.; Thorpe, G.C.; Moore, H.J.; Deane, K.H.; AlAbdulghafoor, F.K.; Summerbell, C.D.; Worthington, H.V.; et al. Omega-3 fatty acids for the primary and secondary prevention of cardiovascular disease. Cochrane Database Syst. Rev. 2018, 7, CD003177. [CrossRef] [PubMed]

14. Hu, Y.; Hu, F.B.; Manson, J.E. Marine omega-3 supplementation and cardiovascular disease: An updated meta-analysis of 13 randomized controlled trials involving 127,477 participants. J. Am. Heart Assoc. 2019, 8, e013543. [CrossRef] [PubMed]

15. Hoang, T.; Kim, J. Comparative effect of statins and omega-3 supplementation on cardiovascular events: Meta-analysis and network meta-analysis of 63 randomized controlled trials including 264,516 participants. Nutrients 2020, 12, 2218. [CrossRef] [PubMed] 
16. DerSimonian, R.; Laird, N. Meta-analysis in clinical trials. Control. Clin. Trials 1986, 7, 177-188. [CrossRef]

17. Shim, S.; Yoon, B.H.; Shin, I.S.; Bae, J.M. Network meta-analysis: Application and practice using Stata. Epidemiol. Health 2017, 39, e2017047. [CrossRef]

18. Higgins, J.P.; Thompson, S.G.; Deeks, J.J.; Altman, D.G. Measuring inconsistency in meta-analyses. BMJ 2003, 327, 557-560. [CrossRef]

19. Sterne, J.A.; Egger, M. Funnel plots for detecting bias in meta-analysis: Guidelines on choice of axis. J. Clin. Epidemiol. 2001, 54, 1046-1055. [CrossRef]

20. Egger, M.; Davey Smith, G.; Schneider, M.; Minder, C. Bias in meta-analysis detected by a simple, graphical test. BMJ 1997, 315, 629-634. [CrossRef]

21. Sterne, J.A.; Sutton, A.J.; Ioannidis, J.P.; Terrin, N.; Jones, D.R.; Lau, J.; Carpenter, J.; Rucker, G.; Harbord, R.M.; Schmid, C.H.; et al. Recommendations for examining and interpreting funnel plot asymmetry in meta-analyses of randomised controlled trials. BMJ 2011, 343, d4002. [CrossRef]

22. Orsini, N.; Li, R.; Wolk, A.; Khudyakov, P.; Spiegelman, D. Meta-analysis for linear and nonlinear dose-response relations: Examples, an evaluation of approximations, and software. Am. J. Epidemiol. 2012, 175, 66-73. [CrossRef] [PubMed]

23. Andrieu, S.; Guyonnet, S.; Coley, N.; Cantet, C.; Bonnefoy, M.; Bordes, S.; Bories, L.; Cufi, M.N.; Dantoine, T.; Dartigues, J.F.; et al. Effect of long-term omega 3 polyunsaturated fatty acid supplementation with or without multidomain intervention on cognitive function in elderly adults with memory complaints (MAPT): A randomised, placebo-controlled trial. Lancet Neurol. 2017, 16, 377-389. [CrossRef]

24. Ascend Study Collaborative Group; Bowman, L.; Mafham, M.; Wallendszus, K.; Stevens, W.; Buck, G.; Barton, J.; Murphy, K.; Aung, T.; Haynes, R.; et al. Effects of n-3 fatty acid supplements in diabetes mellitus. N. Engl. J. Med. 2018, 379, 1540-1550. [CrossRef] [PubMed]

25. Asselbergs, F.W.; Diercks, G.F.; Hillege, H.L.; van Boven, A.J.; Janssen, W.M.; Voors, A.A.; de Zeeuw, D.; de Jong, P.E.; van Veldhuisen, D.J.; van Gilst, W.H.; et al. Effects of fosinopril and pravastatin on cardiovascular events in subjects with microalbuminuria. Circulation 2004, 110, 2809-2816. [CrossRef]

26. Athyros, V.G.; Papageorgiou, A.A.; Mercouris, B.R.; Athyrou, V.V.; Symeonidis, A.N.; Basayannis, E.O.; Demitriadis, D.S.; Kontopoulos, A.G. Treatment with atorvastatin to the National Cholesterol Educational Program goal versus 'usual' care in secondary coronary heart disease prevention. The GREek Atorvastatin and Coronary-heart-disease Evaluation (GREACE) study. Curr. Med. Res. Opin. 2002, 18, 220-228. [CrossRef]

27. Bestehorn, H.P.; Rensing, U.F.; Roskamm, H.; Betz, P.; Benesch, L.; Schemeitat, K.; Blumchen, G.; Claus, J.; Mathes, P.; Kappenberger, L.; et al. The effect of simvastatin on progression of coronary artery disease. The Multicenter coronary Intervention Study (CIS). Eur. Heart J. 1997, 18, 226-234. [CrossRef]

28. Bhatt, D.L.; Steg, P.G.; Miller, M.; Brinton, E.A.; Jacobson, T.A.; Ketchum, S.B.; Doyle, R.T., Jr.; Juliano, R.A.; Jiao, L.; Granowitz, C.; et al. Cardiovascular risk reduction with icosapent ethyl for hypertriglyceridemia. N. Engl. J. Med. 2019, 380, 11-22. [CrossRef]

29. Blankenhorn, D.H.; Azen, S.P.; Kramsch, D.M.; Mack, W.J.; Cashin-Hemphill, L.; Hodis, H.N.; DeBoer, L.W.; Mahrer, P.R.; Masteller, M.J.; Vailas, L.I.; et al. Coronary angiographic changes with lovastatin therapy. The Monitored Atherosclerosis Regression Study (MARS). Ann. Intern. Med. 1993, 119, 969-976. [CrossRef]

30. Brouwer, I.A.; Zock, P.L.; Camm, A.J.; Bocker, D.; Hauer, R.N.; Wever, E.F.; Dullemeijer, C.; Ronden, J.E.; Katan, M.B.; Lubinski, A.; et al. Effect of fish oil on ventricular tachyarrhythmia and death in patients with implantable cardioverter defibrillators: The Study on Omega-3 Fatty Acids and Ventricular Arrhythmia (SOFA) randomized trial. JAMA 2006, 295, 2613-2619. [CrossRef]

31. Chan, K.L.; Teo, K.; Dumesnil, J.G.; Ni, A.; Tam, J.; Investigators, A. Effect of lipid lowering with rosuvastatin on progression of aortic stenosis: Results of the aortic stenosis progression observation: Measuring effects of rosuvastatin (ASTRONOMER) trial. Circulation 2010, 121, 306-314. [CrossRef]

32. Colhoun, H.M.; Betteridge, D.J.; Durrington, P.N.; Hitman, G.A.; Neil, H.A.; Livingstone, S.J.; Thomason, M.J.; Mackness, M.I.; Charlton-Menys, V.; Fuller, J.H.; et al. Primary prevention of cardiovascular disease with atorvastatin in type 2 diabetes in the Collaborative Atorvastatin Diabetes Study (CARDS): Multicentre randomised placebo-controlled trial. Lancet 2004, 364, 685-696. [CrossRef]

33. Dangour, A.D.; Allen, E.; Elbourne, D.; Fasey, N.; Fletcher, A.E.; Hardy, P.; Holder, G.E.; Knight, R.; Letley, L.; Richards, M.; et al. Effect of 2-y n-3 long-chain polyunsaturated fatty acid supplementation on cognitive function in older people: A randomized, double-blind, controlled trial. Am. J. Clin. Nutr. 2010, 91, 1725-1732. [CrossRef] 
34. Downs, J.R.; Clearfield, M.; Weis, S.; Whitney, E.; Shapiro, D.R.; Beere, P.A.; Langendorfer, A.; Stein, E.A.; Kruyer, W.; Gotto, A.M., Jr. Primary prevention of acute coronary events with lovastatin in men and women with average cholesterol levels: Results of AFCAPS/TexCAPS. Air Force/Texas Coronary Atherosclerosis Prevention Study. JAMA 1998, 279, 1615-1622. [CrossRef] [PubMed]

35. Einvik, G.; Klemsdal, T.O.; Sandvik, L.; Hjerkinn, E.M. A randomized clinical trial on n-3 polyunsaturated fatty acids supplementation and all-cause mortality in elderly men at high cardiovascular risk. Eur. J. Cardiovasc. Prev. Rehabil. 2010, 17, 588-592. [CrossRef] [PubMed]

36. Ford, I.; Murray, H.; McCowan, C.; Packard, C.J. Long-term safety and efficacy of lowering low-density lipoprotein cholesterol with statin therapy: 20-year follow-up of West of Scotland Coronary Prevention Study. Circulation 2016, 133, 1073-1080. [CrossRef]

37. Furberg, C.D.; Adams, H.P., Jr.; Applegate, W.B.; Byington, R.P.; Espeland, M.A.; Hartwell, T.; Hunninghake, D.B.; Lefkowitz, D.S.; Probstfield, J.; Riley, W.A.; et al. Effect of lovastatin on early carotid atherosclerosis and cardiovascular events. Asymptomatic Carotid Artery Progression Study (ACAPS) Research Group. Circulation 1994, 90, 1679-1687. [CrossRef]

38. Furberg, C.D.; Pitt, B.; Byington, R.P.; Park, J.S.; McGovern, M.E. Reduction in coronary events during treatment with pravastatin. PLAC I and PLAC II Investigators. Pravastatin Limitation of Atherosclerosis in the Coronary Arteries. Am. J. Cardiol. 1995, 76, 60C-63C. [CrossRef]

39. Galan, P.; Kesse-Guyot, E.; Czernichow, S.; Briancon, S.; Blacher, J.; Hercberg, S.; SU.FOL.OM3 Collaborative Group. Effects of B vitamins and omega 3 fatty acids on cardiovascular diseases: A randomised placebo controlled trial. BMJ 2010, 341, c6273. [CrossRef]

40. Gissi, P.I. Dietary supplementation with n-3 polyunsaturated fatty acids and vitamin E after myocardial infarction: Results of the GISSI-Prevenzione trial. Gruppo Italiano per lo Studio della Sopravvivenza nell'Infarto miocardico. Lancet 1999, 354, 447-455.

41. Jonathan, E.; Derrick, B.; Emma, L.; Sarah, P.; John, D.; Jane, A.; Rory, C.; Heart Protection Study Collaborative Group. C-reactive protein concentration and the vascular benefits of statin therapy: An analysis of 20,536 patients in the Heart Protection Study. Lancet 2011, 377, 469-476. [CrossRef]

42. Investigators, O.T.; Bosch, J.; Gerstein, H.C.; Dagenais, G.R.; Diaz, R.; Dyal, L.; Jung, H.; Maggiono, A.P.; Probstfield, J.; Ramachandran, A.; et al. n-3 fatty acids and cardiovascular outcomes in patients with dysglycemia. N. Engl. J. Med. 2012, 367, 309-318. [CrossRef]

43. Kjekshus, J.; Apetrei, E.; Barrios, V.; Bohm, M.; Cleland, J.G.; Cornel, J.H.; Dunselman, P.; Fonseca, C.; Goudev, A.; Grande, P.; et al. Rosuvastatin in older patients with systolic heart failure. N. Engl. J. Med. 2007, 357, 2248-2261. [CrossRef] [PubMed]

44. Knopp, R.H.; D'Emden, M.; Smilde, J.G.; Pocock, S.J. Efficacy and safety of atorvastatin in the prevention of cardiovascular end points in subjects with type 2 diabetes: The Atorvastatin Study for Prevention of Coronary Heart Disease Endpoints in non-insulin-dependent diabetes mellitus (ASPEN). Diabetes Care 2006, 29, 1478-1485. [CrossRef] [PubMed]

45. Koren, M.J.; Hunninghake, D.B.; Investigators, A. Clinical outcomes in managed-care patients with coronary heart disease treated aggressively in lipid-lowering disease management clinics: The alliance study. J. Am. Coll Cardiol. 2004, 44, 1772-1779. [CrossRef]

46. Kromhout, D.; Giltay, E.J.; Geleijnse, J.M.; Alpha Omega Trial, G. n-3 fatty acids and cardiovascular events after myocardial infarction. N. Engl. J. Med. 2010, 363, 2015-2026. [CrossRef]

47. Liem, A.H.; van Boven, A.J.; Veeger, N.J.; Withagen, A.J.; Robles de Medina, R.M.; Tijssen, J.G.; van Veldhuisen, D.J. Effect of fluvastatin on ischaemia following acute myocardial infarction: A randomized trial. Eur. Heart J. 2002, 23, 1931-1937. [CrossRef]

48. Long-Term Intervention with Pravastatin in Ischaemic Disease (LIPID) Study Group. Prevention of cardiovascular events and death with pravastatin in patients with coronary heart disease and a broad range of initial cholesterol levels. N. Engl. J. Med. 1998, 339, 1349-1357. [CrossRef] [PubMed]

49. Macchia, A.; Grancelli, H.; Varini, S.; Nul, D.; Laffaye, N.; Mariani, J.; Ferrante, D.; Badra, R.; Figal, J.; Ramos, S.; et al. Omega-3 fatty acids for the prevention of recurrent symptomatic atrial fibrillation: Results of the FORWARD (Randomized Trial to Assess Efficacy of PUFA for the Maintenance of Sinus Rhythm in Persistent Atrial Fibrillation) trial. J. Am. Coll. Cardiol. 2013, 61, 463-468. [CrossRef] [PubMed] 
50. Makuuchi, H.; Furuse, A.; Endo, M.; Nakamura, H.; Daida, H.; Watanabe, M.; Ohashi, Y.; Hosoda, Y.; Hosoda, S.; Yamaguchi, H.; et al. Effect of pravastatin on progression of coronary atherosclerosis in patients after coronary artery bypass surgery. Circ. J. 2005, 69, 636-643. [CrossRef]

51. Manson, J.E.; Cook, N.R.; Lee, I.M.; Christen, W.; Bassuk, S.S.; Mora, S.; Gibson, H.; Albert, C.M.; Gordon, D.; Copeland, T.; et al. Marine n-3 fatty acids and prevention of cardiovascular disease and cancer. N. Engl. J. Med. 2019, 380, 23-32. [CrossRef]

52. Nakagawa, T.; Kobayashi, T.; Awata, N.; Sato, S.; Reiber, J.H.; Nakajima, H.; Toyama, Y.N.; Hiraoka, H.; Kato, O.; Kirino, M.; et al. Randomized, controlled trial of secondary prevention of coronary sclerosis in normocholesterolemic patients using pravastatin: Final 5-year angiographic follow-up of the Prevention of Coronary Sclerosis (PCS) study. Int. J. Cardiol. 2004, 97, 107-114. [CrossRef] [PubMed]

53. Nakamura, H.; Arakawa, K.; Itakura, H.; Kitabatake, A.; Goto, Y.; Toyota, T.; Nakaya, N.; Nishimoto, S.; Muranaka, M.; Yamamoto, A.; et al. Primary prevention of cardiovascular disease with pravastatin in Japan (MEGA Study): A prospective randomised controlled trial. Lancet 2006, 368, 1155-1163. [CrossRef]

54. Nilsen, D.W.; Albrektsen, G.; Landmark, K.; Moen, S.; Aarsland, T.; Woie, L. Effects of a high-dose concentrate of n-3 fatty acids or corn oil introduced early after an acute myocardial infarction on serum triacylglycerol and HDL cholesterol. Am. J. Clin. Nutr. 2001, 74, 50-56. [CrossRef] [PubMed]

55. ALLHAT Collaborative Research Group. Major outcomes in moderately hypercholesterolemic, hypertensive patients randomized to pravastatin vs usual care: The Antihypertensive and Lipid-Lowering Treatment to Prevent Heart Attack Trial (ALLHAT-LLT). JAMA 2002, 288, 2998-3007. [CrossRef] [PubMed]

56. Ostadal, P.; Alan, D.; Vejvoda, J.; Kukacka, J.; Macek, M.; Hajek, P.; Mates, M.; Kvapil, M.; Kettner, J.; Wiendl, M.; et al. Fluvastatin in the first-line therapy of acute coronary syndrome: Results of the multicenter, randomized, double-blind, placebo-controlled trial (the FACS-trial). Trials 2010, 11, 61. [CrossRef] [PubMed]

57. Pedersen, T.R.; Faergeman, O.; Kastelein, J.J.; Olsson, A.G.; Tikkanen, M.J.; Holme, I.; Larsen, M.L.; Bendiksen, F.S.; Lindahl, C.; Szarek, M.; et al. High-dose atorvastatin vs usual-dose simvastatin for secondary prevention after myocardial infarction: The IDEAL study: A randomized controlled trial. JAMA 2005, 294, 2437-2445. [CrossRef]

58. Raitt, M.H.; Connor, W.E.; Morris, C.; Kron, J.; Halperin, B.; Chugh, S.S.; McClelland, J.; Cook, J.; MacMurdy, K.; Swenson, R.; et al. Fish oil supplementation and risk of ventricular tachycardia and ventricular fibrillation in patients with implantable defibrillators: A randomized controlled trial. JAMA 2005, 293, 2884-2891. [CrossRef]

59. Rauch, B.; Schiele, R.; Schneider, S.; Diller, F.; Victor, N.; Gohlke, H.; Gottwik, M.; Steinbeck, G.; Del Castillo, U.; Sack, R.; et al. OMEGA, a randomized, placebo-controlled trial to test the effect of highly purified omega-3 fatty acids on top of modern guideline-adjusted therapy after myocardial infarction. Circulation 2010, 122, 2152-2159. [CrossRef]

60. Ridker, P.M.; Danielson, E.; Fonseca, F.A.; Genest, J.; Gotto, A.M., Jr.; Kastelein, J.J.; Koenig, W.; Libby, P.; Lorenzatti, A.J.; MacFadyen, J.G.; et al. Rosuvastatin to prevent vascular events in men and women with elevated C-reactive protein. N. Engl. J. Med. 2008, 359, 2195-2207. [CrossRef]

61. Riegger, G.; Abletshauser, C.; Ludwig, M.; Schwandt, P.; Widimsky, J.; Weidinger, G.; Welzel, D. The effect of fluvastatin on cardiac events in patients with symptomatic coronary artery disease during one year of treatment. Atherosclerosis 1999, 144, 263-270. [CrossRef]

62. Roncaglioni, M.C.; Tombesi, M.; Avanzini, F.; Barlera, S.; Caimi, V.; Longoni, P.; Marzona, I.; Milani, V.; Silletta, M.G.; Risk and Prevention Study Collaborative Group; et al. n-3 fatty acids in patients with multiple cardiovascular risk factors. N. Engl. J. Med. 2013, 368, 1800-1808. [CrossRef] [PubMed]

63. Sawayama, Y.; Shimizu, C.; Maeda, N.; Tatsukawa, M.; Kinukawa, N.; Koyanagi, S.; Kashiwagi, S.; Hayashi, J. Effects of probucol and pravastatin on common carotid atherosclerosis in patients with asymptomatic hypercholesterolemia. Fukuoka Atherosclerosis Trial (FAST). J. Am. Coll. Cardiol. 2002, 39, 610-616. [CrossRef] 
64. Serruys, P.W.; de Feyter, P.; Macaya, C.; Kokott, N.; Puel, J.; Vrolix, M.; Branzi, A.; Bertolami, M.C.; Jackson, G.; Strauss, B.; et al. Fluvastatin for prevention of cardiac events following successful first percutaneous coronary intervention: A randomized controlled trial. JAMA 2002, 287, 3215-3222. [CrossRef]

65. Sever, P.S.; Dahlof, B.; Poulter, N.R.; Wedel, H.; Beevers, G.; Caulfield, M.; Collins, R.; Kjeldsen, S.E.; Kristinsson, A.; McInnes, G.T.; et al. Prevention of coronary and stroke events with atorvastatin in hypertensive patients who have average or lower-than-average cholesterol concentrations, in the Anglo-Scandinavian Cardiac Outcomes Trial-Lipid Lowering Arm (ASCOT-LLA): A multicentre randomised controlled trial. Drugs 2004, 64 (Suppl. 2), 43-60. [CrossRef]

66. Shepherd, J.; Blauw, G.J.; Murphy, M.B.; Bollen, E.L.; Buckley, B.M.; Cobbe, S.M.; Ford, I.; Gaw, A.; Hyland, M.; Jukema, J.W.; et al. Pravastatin in elderly individuals at risk of vascular disease (PROSPER): A randomised controlled trial. Lancet 2002, 360, 1623-1630. [CrossRef]

67. Karam, J.G.; Loney-Hutchinson, L.; McFarlane, S.I.; Stroke Prevention by Aggressive Reduction in Cholesterol Levels (SPARCL) Investigators. High-dose atorvastatin after stroke or transient ischemic attack: The Stroke Prevention by Aggressive Reduction in Cholesterol Levels (SPARCL) Investigators. N. Engl. J. Med. 2008, 3, 68-69. [CrossRef] [PubMed]

68. Takano, H.; Mizuma, H.; Kuwabara, Y.; Sato, Y.; Shindo, S.; Kotooka, N.; Fujimatsu, D.; Kobayashi, Y.; Inoue, T.; Node, K.; et al. Effects of pitavastatin in Japanese patients with chronic heart failure: The Pitavastatin Heart Failure Study (PEARL Study). Circ. J. 2013, 77, 917-925. [CrossRef] [PubMed]

69. Tavazzi, L.; Maggioni, A.P.; Marchioli, R.; Barlera, S.; Franzosi, M.G.; Latini, R.; Lucci, D.; Nicolosi, G.L.; Porcu, M.; Tognoni, G.; et al. Effect of n-3 polyunsaturated fatty acids in patients with chronic heart failure (the GISSI-HF trial): A randomised, double-blind, placebo-controlled trial. Lancet 2008, 372, 1223-1230. [CrossRef]

70. Tavazzi, L.; Maggioni, A.P.; Marchioli, R.; Barlera, S.; Franzosi, M.G.; Latini, R.; Lucci, D.; Nicolosi, G.L.; Porcu, M.; Tognoni, G.; et al. Effect of rosuvastatin in patients with chronic heart failure (the GISSI-HF trial): A randomised, double-blind, placebo-controlled trial. Lancet 2008, 372, 1231-1239. [CrossRef]

71. Teo, K.K.; Burton, J.R.; Buller, C.E.; Plante, S.; Catellier, D.; Tymchak, W.; Dzavik, V.; Taylor, D.; Yokoyama, S.; Montague, T.J. Long-term effects of cholesterol lowering and angiotensin-converting enzyme inhibition on coronary atherosclerosis: The Simvastatin/Enalapril Coronary Atherosclerosis Trial (SCAT). Circulation 2000, 102, 1748-1754. [CrossRef]

72. Wanner, C.; Krane, V.; Marz, W.; Olschewski, M.; Mann, J.F.; Ruf, G.; Ritz, E.; German, D.; German Diabetes and Dialysis Study Investigators. Atorvastatin in patients with type 2 diabetes mellitus undergoing hemodialysis. N. Engl. J. Med. 2005, 353, 238-248. [CrossRef] [PubMed]

73. Bonds, D.E.; Harrington, M.; Worrall, B.B.; Bertoni, A.G.; Eaton, C.B.; Hsia, J.; Robinson, J.; Clemons, T.E.; Fine, L.J.; Writing Group for the AREDS2 Research Group; et al. Effect of long-chain omega-3 fatty acids and lutein + zeaxanthin supplements on cardiovascular outcomes: Results of the Age-Related Eye Disease Study 2 (AREDS2) randomized clinical trial. JAMA Intern. Med. 2014, 174, 763-771. [CrossRef] [PubMed]

74. Yokoi, H.; Nobuyoshi, M.; Mitsudo, K.; Kawaguchi, A.; Yamamoto, A.; Investigators, A.S. Three-year follow-up results of angiographic intervention trial using an HMG-CoA reductase inhibitor to evaluate retardation of obstructive multiple atheroma (ATHEROMA) study. Circ. J. 2005, 69, 875-883. [CrossRef] [PubMed]

75. Yokoyama, M.; Origasa, H.; Matsuzaki, M.; Matsuzawa, Y.; Saito, Y.; Ishikawa, Y.; Oikawa, S.; Sasaki, J.; Hishida, H.; Itakura, H.; et al. Effects of eicosapentaenoic acid on major coronary events in hypercholesterolaemic patients (JELIS): A randomised open-label, blinded endpoint analysis. Lancet 2007, 369, 1090-1098. [CrossRef]

76. Yusuf, S.; Bosch, J.; Dagenais, G.; Zhu, J.; Xavier, D.; Liu, L.; Pais, P.; Lopez-Jaramillo, P.; Leiter, L.A.; Dans, A.; et al. Cholesterol Lowering in Intermediate-Risk Persons without Cardiovascular Disease. N. Engl. J. Med. 2016, 374, 2021-2031. [CrossRef]

77. Pfizer. Lipitor (Atorvastatin): Highlights of Prescribing Information. Available online: https://www. accessdata.fda.gov/drugsatfda_docs/label/2009/020702s056lbl.pdf (accessed on 7 April 2020).

78. Novartis. Lescol (Fluvastatin): Highlights of Prescribing Information. Available online: https://www. accessdata.fda.gov/drugsatfda_docs/label/2012/021192s019lbl.pdf (accessed on 7 April 2020). 
79. Watson Laboratories. Altoprev (Lovastatin Extended-Release): Highlights of Prescribing Information. Available online: https://www.accessdata.fda.gov/drugsatfda_docs/label/2012/021316s028lbl.pdf (accessed on 7 April 2020).

80. Kowa. Livalo (Pitavastatin): Highlights of Prescribing Information. Available online: https://www.accessdata. fda.gov/drugsatfda_docs/label/2019/022363s015lbl.pdf (accessed on 7 April 2020).

81. AstraZeneca. Crestor (Rosuvastatin Calcium): Highlights of Prescribing Information. Available online: https://www.accessdata.fda.gov/drugsatfda_docs/label/2010/021366s016lbl.pdf (accessed on 7 April 2020).

82. Marck \& Co. Zocor (Simvastatin): Highlights of Prescribing Information. Available online: https: //www.accessdata.fda.gov/drugsatfda_docs/label/2010/019766s078lbl.pdf (accessed on 7 April 2020).

83. Bristol-Myers Squibb. Pravachol (Pravastatin Sodium): Highlights of Prescribing Information. Available online: https://www.accessdata.fda.gov/drugsatfda_docs/label/2012/019898s062lbl.pdf (accessed on 7 April 2020).

84. Scandinavian Simvastatin Survival Study Group. Randomised trial of cholesterol lowering in 4444 patients with coronary heart disease: The Scandinavian Simvastatin Survival Study (4S). Lancet 1994, 344, 1383-1389.

85. An, K.; Huang, R.; Tian, S.; Guo, D.; Wang, J.; Lin, H.; Wang, S. Statins significantly reduce mortality in patients receiving clopidogrel without affecting platelet activation and aggregation: A systematic review and meta-analysis. Lipids Health Dis. 2019, 18, 121. [CrossRef]

86. Chen, P.H.; Wang, J.S.; Lin, S.Y.; Li, C.H.; Wang, C.Y.; Hu, C.Y.; Fu, C.P.; Tsai, Y.T.; Lee, A.T.; Chen, Y.W.; et al. Effects of statins on all-cause mortality at different low-density-lipoprotein cholesterol levels in Asian patients with type 2 diabetes. Curr. Med. Res. Opin. 2018, 34, 1885-1892. [CrossRef]

87. Lipworth, L.; Fazio, S.; Kabagambe, E.K.; Munro, H.M.; Nwazue, V.C.; Tarone, R.E.; McLaughlin, J.K.; Blot, W.J.; Sampson, U.K. A prospective study of statin use and mortality among 67,385 blacks and whites in the Southeastern United States. Clin. Epidemiol. 2014, 6, 15-25. [CrossRef]

88. Tada, H.; Nohara, A.; Kawashiri, M.A. Serum Triglycerides and Atherosclerotic Cardiovascular Disease: Insights from Clinical and Genetic Studies. Nutrients 2018, 10, 1789. [CrossRef]

89. Jeong, S.M.; Choi, S.; Kim, K.; Kim, S.M.; Lee, G.; Park, S.Y.; Kim, Y.Y.; Son, J.S.; Yun, J.M.; Park, S.M. Effect of Change in Total Cholesterol Levels on Cardiovascular Disease Among Young Adults. J. Am. Heart Assoc. 2018, 7. [CrossRef]

90. Choi, S. The potential role of biomarkers associated with ASCVD risk: Risk-enhancing biomarkers. J. Lipid Atheroscler. 2019, 8, 173-182. [CrossRef] [PubMed]

91. Kim, J.; Hoang, T.; Bu, S.Y.; Kim, J.M.; Choi, J.H.; Park, E.; Lee, S.M.; Park, E.; Min, J.Y.; Lee, I.S.; et al. Association of dietary intake with cardiovascular disease, blood pressure, and lipid profile in the Korean population: A systematic review and meta-analysis. J. Lipid Atheroscler. 2020, 9, 205-229. [CrossRef]

92. Doshi, R.; Kumar, A.; Thakkar, S.; Shariff, M.; Adalja, D.; Doshi, A.; Taha, M.; Gupta, R.; Desai, R.; Shah, J.; et al. Meta-analysis comparing combined use of eicosapentaenoic acid and statin to statin alone. Am. J. Cardiol. 2020, 125, 198-204. [CrossRef] [PubMed]

93. Oscarsson, J.; Hurt-Camejo, E. Omega-3 fatty acids eicosapentaenoic acid and docosahexaenoic acid and their mechanisms of action on apolipoprotein B-containing lipoproteins in humans: A review. Lipids Health Dis. 2017, 16, 149. [CrossRef]

94. Qin, Y.; Zhou, Y.; Chen, S.H.; Zhao, X.L.; Ran, L.; Zeng, X.L.; Wu, Y.; Chen, J.L.; Kang, C.; Shu, F.R.; et al. Fish Oil Supplements Lower Serum Lipids and Glucose in Correlation with a Reduction in Plasma Fibroblast Growth Factor 21 and Prostaglandin E2 in Nonalcoholic Fatty Liver Disease Associated with Hyperlipidemia: A Randomized Clinical Trial. PLoS ONE 2015, 10, e0133496. [CrossRef]

95. Jeong, G.H.; Lee, K.H.; Kim, J.Y.; Eisenhut, M.; Kronbichler, A.; van der Vliet, H.J.; Shin, J.I.; Gamerith, G. Statin and cancer mortality and survival: An umbrella systematic review and meta-analysis. J. Clin. Med. 2020, 9, 326. [CrossRef]

96. Bird, J.K.; Calder, P.C.; Eggersdorfer, M. The Role of n-3 Long Chain Polyunsaturated Fatty Acids in Cardiovascular Disease Prevention, and Interactions with Statins. Nutrients 2018, 10, 775. [CrossRef] [PubMed]

97. Choi, H.D.; Chae, S.M. Comparison of efficacy and safety of combination therapy with statins and omega-3 fatty acids versus statin monotherapy in patients with dyslipidemia: A systematic review and meta-analysis. Medicine 2018, 97, e13593. [CrossRef] 
98. Kim, C.H.; Han, K.A.; Yu, J.; Lee, S.H.; Jeon, H.K.; Kim, S.H.; Kim, S.Y.; Han, K.H.; Won, K.; Kim, D.B.; et al. Efficacy and Safety of Adding Omega-3 Fatty Acids in Statin-treated Patients with Residual Hypertriglyceridemia: ROMANTIC (Rosuvastatin-OMAcor iN residual hyperTrIglyCeridemia), a Randomized, Double-blind, and Placebo-controlled Trial. Clin. Ther. 2018, 40, 83-94. [CrossRef]

99. Jun, J.E.; Jeong, I.K.; Yu, J.M.; Kim, S.R.; Lee, I.K.; Han, K.A.; Choi, S.H.; Kim, S.K.; Park, H.K.; Mok, J.O.; et al. Efficacy and Safety of Omega-3 Fatty Acids in Patients Treated with Statins for Residual Hypertriglyceridemia: A Randomized, Double-Blind, Placebo-Controlled Clinical Trial. Diabetes Metab. J. 2020, 44, 78-90. [CrossRef] [PubMed]

Publisher's Note: MDPI stays neutral with regard to jurisdictional claims in published maps and institutional affiliations.

(C) 2020 by the authors. Licensee MDPI, Basel, Switzerland. This article is an open access article distributed under the terms and conditions of the Creative Commons Attribution (CC BY) license (http://creativecommons.org/licenses/by/4.0/). 